\title{
Curcumin-loaded redox response of self- assembled micelles for enhanced antitumor and anti-inflammation efficacy
}

This article was published in the following Dove Press journal:

International Journal of Nanomedicine

29 March 2017

Number of times this article has been viewed

\section{Shuang Zhao',* \\ Litao Ma',* \\ Chengwen Cao' \\ Qianqian $\mathrm{Yu}^{\prime}$ \\ Lanmei Chen ${ }^{1,2}$ \\ Jie Liu'}

'Department of Chemistry, Jinan University, Guangzhou, ${ }^{2}$ Department of Chemistry, School of Pharmacy, Guangdong Medical University,

Zhanjiang, People's Republic of China

*These authors contributed equally to this work
Correspondence: Lanmei Chen Department of Chemistry, School of Pharmacy, Guangdong Medical University, No 2, Wenming East Road, Zhanjiang 524023, People's Republic of China Tel/fax +86 2085220223

Email lanmeichen@।26.com

Jie Liu

Department of Chemistry, Jinan University, No 60I, Huangpu

Avenue West, Guangzhou 510632,

People's Republic of China

Tel/fax +86 2085220223

Email tliuliu@jnu.edu.cn
Abstract: At present, it has become evident that inflammation plays a critical role in tumor growth; meanwhile, chemotherapeutic agents using nanocarriers have been suggested as a promising strategy in cancer treatment. In this study, novel redox-responsive micelles were prepared from monomethoxy-poly(ethylene glycol)-chitosan-S-S-hexadecyl $\left(\mathrm{C}_{16}-\mathrm{SS}\right.$ CS-mPEG). These micelles were able to carry and deliver drugs into tumor cells. To serve as a control, monomethoxy-poly(ethylene glycol)-chitosan-C-C-hexadecyl $\left(\mathrm{C}_{16}\right.$-CC-CSmPEG) was developed in a similar fashion to that used to yield $\mathrm{C}_{16}$-CC-CS-mPEG without a redox-responsive disulfide bond. The cellular uptake mechanisms of both micelles were determined. The efficient intracellular drug release from micelles in MCF-7 cells was further confirmed. Results indicated that curcumin (Cur) could rapidly form $\mathrm{C}_{16}$-SS-CS-mPEG@, Cur micelles when exposed to reducing agents and efficaciously enhance intracellular accumulation. The cytotoxicity assay demonstrated that $\mathrm{C}_{16}$-SS-CS-mPEG@Cur exhibited satisfactory cytotoxicity against MCF-7 cells. Anti-inflammation assay results indicated that $\mathrm{C}_{16}$-SS-CS-mPEG@Cur treatment significantly downregulated tumor necrosis factor (TNF- $\alpha$ ) expression and showed good anti-inflammatory effects in tumor microenvironment. Most importantly, antitumor effects in vivo showed satisfactory therapeutic effects with $\mathrm{C}_{16}$-SS-CS-mPEG@Cur. Hence, $\mathrm{C}_{16}$-SS-CS-mPEG@Cur micelles can be useful in tumor therapy.

Keywords: micelles, curcumin, anti-inflammatory effect, anti-tumor effect, tumor

\section{Introduction}

There is clear evidence that an inflammatory microenvironment is an essential component of all tumors, including some in which a direct causal relationship with inflammation is not yet proven. ${ }^{1-3}$ Obvious inflammation is a key factor in tumor formation, growth, and metastasis. ${ }^{4,5}$ These connections between cancer and inflammation inspire us to combine the treatment of inflammation and cancer to achieve better cancer therapeutic effects. For example, Marques et $\mathrm{al}^{6}$ reported application of nonsteroidal anti-inflammatory drugs in breast cancer therapy.

Recent studies show that a polymeric micelle is one of the most attractive nanocarriers for drugs to avoid their weaknesses such as poor water solubility and improve targeting through the enhanced permeability and retention (EPR) effect. ${ }^{7,8}$ Compared with other delivery systems, polymeric micelles confer some important advantages such as protecting drugs from adverse surrounding environments through entrapping these hydrophobic drugs into a hydrophobic inner core and a uniquely designed structure response to several individual factors in cancer cells, such as low $\mathrm{pH}$ and 
a redox environment. ${ }^{7-9}$ In the present study, chitosan (CS) was chosen as the backbone of the polymeric micelle. Monomethoxy-poly (ethylene glycol), $\mathrm{C}_{16}-\mathrm{SS}-\mathrm{COOH}$, or $\mathrm{C}_{16}{ }^{-}$ $\mathrm{CC}-\mathrm{COOH}$ has been grafted onto $\mathrm{CS}$ to form hydrophobic and hydrophilic structures so that self-assembly into nanosized micellar form was possible. $\mathrm{C}_{16}$-CC-COOH micellar forms were used in contrast with $\mathrm{C}_{16}-\mathrm{SS}-\mathrm{COOH}$ forms to verify redox-responding controlled-release properties caused by glutathione (GSH).

Curcumin (Cur), a polyphenol compound derived from the rhizome of the plant Curcuma longa, exhibits antiinflammatory, anticancer, antioxidant, antimicrobial, and antiangiogenic activities. ${ }^{10-13}$ Among these pharmacological activities, the anticancer and anti-inflammatory activities have attracted the greatest attention because Cur could be a promising chemotherapeutic agent for use in solid tumor therapy. ${ }^{14,15}$ With poor water solubility and an insufficient cellular uptake rate, Cur was limited for further application..$^{16,17}$ Therefore, loading Cur to improve its water solubility and to improve its anticancer and anti-inflammatory effects was desirous. From this standpoint, in this study, we optimized the synthesis of nanosized micelles formulated with different amphiphilic polymers and loaded Cur into micellar forms and examined their antitumor efficacy in vitro and in vivo. The anticancer efficacy of monomethoxy-poly(ethylene glycol)chitosan-S-S-hexadecyl (monomethoxy-poly(ethylene glycol)-chitosan-S-S-hexadecyl with Cur) in recipient mice was evaluated as shown in Scheme 1. Our results indicated that $\mathrm{C}_{16}$-SS-CS-mPEG@Cur had inflammatory microenvironment-enhanced antitumor activity and fewer side effects in vivo, with potential clinical applications.

\section{Materials and methods Materials}

All reagents and solvents were purchased commercially and used without further purification, unless specifically noted. $\mathrm{N}$-Hydroxysuccinimide (NHS), 3,3'-dithiodipropionic acid, 1-hexadecanol, 4-dimethylaminopyridine (DMAP; 99\%, J\&K; Guangdong, People's Republic of China), monomethoxypoly(ethylene glycol) (mPEG; $\mathrm{Mn}=2,000 \mathrm{Da}$, Fluka; St Louis, MO, USA), 1-(3-dimethylaminopropyl)-3-ethylcarbodiimide hydrochloride (EDC·HCl; Shanghai Chemical Co., Shanghai, People's Republic of China), N,N-dimethyl formamide (DMF), and Cur (Shanghai Yingxuan Co., Shanghai, People's Republic of China). MCF-7, HEK293, and NIH/3T3 cells were purchased from American Type Culture Collection (ATCC, Manassas, VA, USA). Fetal bovine serum (FBS) was purchased from Gibco (Life Technologies AG, Switzerland). Ultrapure Milli-Q water (MW 18.2) was used in all experiments.

\section{Synthesis of $\mathrm{C}_{16}$-SS-CS-mPEG and monomethoxy-poly(ethylene glycol)- chitosan-C-C-hexadecyl ( $\mathrm{C}_{16}$-CC-CS- $\mathrm{mPEG)}$}

$\mathrm{C}_{16}$-SS-CS-mPEG was synthesized by a series of reactions. First, under argon atmosphere 3,3'-dithiodipropionic acid $(4.2 \mathrm{~g}, 10 \mathrm{mM})$ and EDC $(4.6 \mathrm{~g}, 11 \mathrm{mM})$ were dissolved in $60 \mathrm{~mL}$ of anhydrous DMF. Then, DMAP (0.48 g, $1 \mathrm{mM})$ and hexadecanol $(9.6 \mathrm{~g}, 10 \mathrm{mM})$ were added to the solution and stirred at $0^{\circ} \mathrm{C}-5^{\circ} \mathrm{C}$ for $2 \mathrm{~h}$, followed by stirring for another $4 \mathrm{~h}$ at $25^{\circ} \mathrm{C}$. Second, $1.0 \mathrm{~g}$ of CS was dissolved in $100 \mathrm{~mL}$ of $1 \%$ acetic acid solution, and then $0.32 \mathrm{~g} \mathrm{C}_{16}$-SS-COOH, $0.62 \mathrm{~g} \mathrm{EDC}$, and $0.12 \mathrm{~g} \mathrm{NHS}$ were added to another beaker, activated for half an hour with $200 \mathrm{~mL}$ of methanol, and then CS acetic acid solution was added dropwise, which was magnetically stirred at $25^{\circ} \mathrm{C}$ for $72 \mathrm{~h}$, and at the end of the reaction, to the reaction flask was added $60 \mathrm{~mL}$ aqueous ammonia, and the product was centrifuged for $5 \mathrm{~min}$ (speed 8,000 rpm); the resultant product was washed 3-5 times by methanol, and then the final product was transferred to a dialysis bag for 7 days and freeze-dried. Third, under a nitrogen atmosphere, $\mathrm{C}_{16}$-SSCS-mPEG polymer was synthesized. Compound $(0.261 \mathrm{~g}$, $0.6 \mathrm{mM}), \mathrm{EDC}(0.136 \mathrm{~g}, 0.66 \mathrm{mM})$, and DMAP $(0.015 \mathrm{~g}$, $0.13 \mathrm{mM}$ ) were added to an $\mathrm{mPEG}$ solution $(0.2 \mathrm{~g}, 0.1 \mathrm{mM})$ in $7 \mathrm{~mL}$ of anhydrous dichloromethane. The product was centrifuged for $5 \mathrm{~min}$ (speed 8,000 rpm), and the resultant product was washed 3-5 times by methanol, and then the final product was transferred to a dialysis bag for 7 days and freeze-dried. The synthesis procedure of $\mathrm{C}_{16}$-CC-CSmPEG polymer was similar to that of $\mathrm{C}_{16}$-SS-CS-mPEG polymer but 3,3-dithiodipropionic acid was replaced with octanedioic acid.

\section{Preparation of Cur-loaded micelles}

Cur-loaded polymeric micelles were prepared by the dialysis method. Before loading Cur into the micelles, $10 \mathrm{mg}$ of Cur was dissolved in $1 \mathrm{~mL}$ of alcohol. Then, $\mathrm{C}_{16}$-SS-CS-mPEG polymer $\left(50 \mathrm{mg}\right.$ ) or $\mathrm{C}_{16}$-CC-CS-mPEG polymer $(50 \mathrm{mg}$ ) was added to the solution, which was stirred at $25^{\circ} \mathrm{C}$ for $24 \mathrm{~h}$. The final mixture was transferred to a dialysis bag (MWCO 8000) and dialyzed against alcohol at $25^{\circ} \mathrm{C}$ for $24 \mathrm{~h}$ to remove the unloaded Cur. The water was exchanged six times during entire procedure. Then, the product was collected by freeze-drying. The Cur concentration in alcohol was determined by fluorescence measurement using a calibration curve constructed from Cur/alcohol solutions with different Cur concentrations. 
A

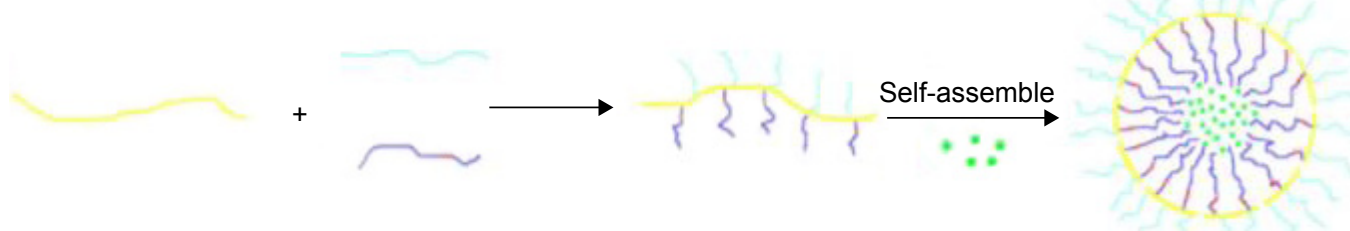

CS $\mathrm{mPEG} \sim \mathrm{C}_{16}$-SS-CS-mPEG $*$ Cur

B Blood vessel

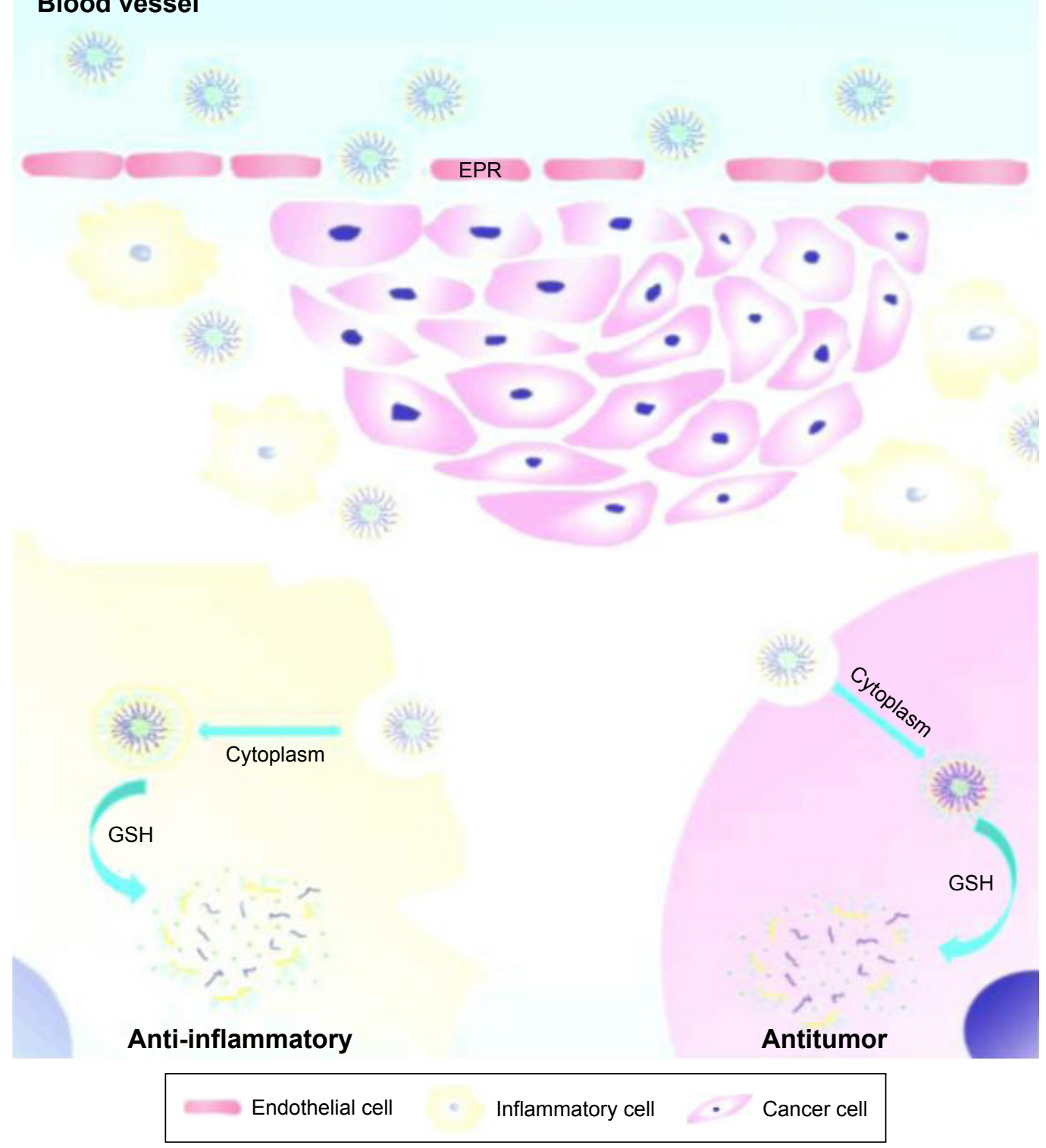

Scheme I Schematic illustration of the preparation of $\mathrm{C}_{16}$-SS-CS-mPEG@Cur micelles as drug delivery system for efficient cancer therapy and inflammatory therapy. Notes: (A) The formation of $\mathrm{C}_{16}$-SS-CS-mPEG@Cur micelles and (B) extracellular and intracellular trafficking for the delivery of Cur to cancer cells and inflammatory cells. Abbreviations: $\mathrm{C}_{16}$-SS-CS-mPEG, monomethoxy-poly(ethylene glycol)-chitosan-S-S-hexadecyl; Cur, curcumin; EPR, enhanced permeability and retention; GSH, glutathione.

\section{Characterization}

Fourier transform-infrared spectrometry (FTIR) samples were recorded using an Equinox 55 IR spectrometer in the range $500-4,000 \mathrm{~cm}^{-1}$ by the $\mathrm{KBr}$-disk method. ${ }^{1} \mathrm{H}$ nuclear magnetic resonance ( ${ }^{1} \mathrm{H}$ NMR) spectra were recorded using a Mercury VX-300 spectrometer at $300 \mathrm{MHz}$ with $\mathrm{D}_{2} \mathrm{O}$ as the solvent and tetramethylsilane as an internal standard. Samples of $\mathrm{C}_{16}$-SS-CS-mPEG and $\mathrm{C}_{16}$-CC-CS-mPEG were 
dropped onto a holey carbon film on copper grids, allowed to dry, and observed by transmission electron microscopy (TEM). The micrographs were obtained using Hitachi (H-7650, Tokyo, Japan) instrument for TEM, operated at an accelerating voltage of $80 \mathrm{kV}$. The size distribution and stability of the nanoparticles were measured using a Zetasizer Nano-ZS particle analyzer (Malvern Instruments Limited) at least three times.

\section{In vitro reduction-triggered release of Cur from Cur-loaded micelles}

Cur was dissolved in water to prepare the stock solution (1 mg/ $\mathrm{mL}$ ); after being sonicated for $5 \mathrm{~min}$ in an ultrasonic bath, the solution was diluted using methanol (aliquot of the standard solution) to reach the final concentration ranging from 0.5 to $2.0 \mu \mathrm{g} / \mathrm{mL}$ (working solution). Water was used as a blank. The fluorescence intensity was measured (420-475 nm, $\mathrm{n}=5$ ). Linearity was evaluated by linear regression analysis, which was calculated by least-squares regression analysis and analysis of variance (ANOVA) test $(\mathrm{a}=0.05)$.

To investigate the action of the Cur-loaded micelles for Cur released. The Cur-loaded micelles of $\mathrm{C}_{16}-\mathrm{SS}-\mathrm{CS}-\mathrm{mPEG}$ and $\mathrm{C}_{16}$-CC-CS-mPEG were dispersed in $10 \mathrm{~mL}$ of phosphatebuffered saline (PBS) (pH 7.4) or PBS with $10 \mathrm{mM}$ dithiothreitol (DTT) at $37^{\circ} \mathrm{C}$. Aliquots $(50 \mathrm{~mL})$ were taken from the suspension at predetermined time intervals and replaced with an equal volume of the fresh medium, the released samples were centrifuged. The concentration of released Cur was determined by measurement of ultraviolet-visible (UV Vis) spectra at $425 \mathrm{~nm}$ based on the standard curve.

\section{Endocytosis inhibition}

MCF-7 cells were seeded with a density of $5 \times 10^{5}$ cells per well and cultured in the six-well plates for $24 \mathrm{~h}$. The cells were pretreated for $30 \mathrm{~min}$ with three different endocytosis inhibitors separately ( $5 \mathrm{mM}$ methyl- $\beta$-cyclodextrin [MBCD], $0.45 \mathrm{mM}$ sucrose, and $5 \mathrm{mM}$ cytochalasin D). Then, the Curloaded $\mathrm{C}_{16}$-SS-CS-mPEG micelles or Cur-loaded $\mathrm{C}_{16}$-CC-CSmPEG micelles were added at a final Cur concentration of $2 \mathrm{mg} / \mathrm{mL}$. After incubation for $2 \mathrm{~h}$, the medium was removed and cells were washed with PBS solution three times. The uptake was analyzed by confocal laser scanning microscope (CLSM) and flow cytometry (FCM).

\section{Intracellular Cur release}

MCF-7 cells were seeded with a density of $5 \times 10^{5}$ cells per well and cultured in the six-well plates for $24 \mathrm{~h}$. Then, the Cur-loaded $\mathrm{C}_{16}$-CC-CS-mPEG micelles or Cur-loaded $\mathrm{C}_{16}$-CC-CS-mPEG micelles were added at a final $\mathrm{Cur}$ concentration of $2 \mathrm{mg} / \mathrm{mL}$. After incubation for 2, 12, 24 or $48 \mathrm{~h}$, the cells were washed with PBS for $30 \mathrm{~min}$ at room temperature. The uptake was analyzed by CLSM and FCM.

\section{In vitro cytotoxicity assays}

The cytotoxicity of the synthesized nanoparticles was assessed using the MTT assay. Briefly, MCF-7 cells were seeded in 96-well plates at $1 \times 10^{4}$ and incubated for $24 \mathrm{~h}$ at $37^{\circ} \mathrm{C}$ before MTT assay. The cells were then treated with various concentrations of $\mathrm{Cur}, \mathrm{C}_{16}-\mathrm{SS}-\mathrm{CS}-\mathrm{mPEG}, \mathrm{C}_{16}-\mathrm{CC}-\mathrm{CS}$ mPEG, $\mathrm{C}_{16}$-SS-CS-mPEG@Cur, and C 16 -CC-CS-mPEG@ Cur for $24 \mathrm{~h}$, respectively. After incubation, cells were treated with MTT solution (final concentration, $0.5 \mathrm{mg} / \mathrm{mL}$ for each well) for $4 \mathrm{~h}$ at $37^{\circ} \mathrm{C}$. The medium was removed carefully and $150 \mathrm{~mL}$ of dimethylsulfoxide (DMSO) was added to each well to dissolve the precipitate. The absorbance was measured on a microplate reader at $570 \mathrm{~nm}$. To determine the cytotoxicities in normal cells of $\mathrm{C}_{16}-\mathrm{SS}-\mathrm{CS}-\mathrm{mPEG}$ and $\mathrm{C}_{16}$-CC-CS-mPEG as carriers, another MTT assay was performed in HEK293 cells, using the same abovementioned methods. All doses of complexes were parallel tested in triplicate, and the data were presented as averages of standard deviations (SDs) of three independent experiments.

\section{TUNEL and DAPI costaining}

For TUNEL and 4',6-diamidino-2-phenylindole (DAPI) staining assays, MCF-7 cells were seeded in six-well plates with a density of $5 \times 10^{5}$ cells per well, then incubated with Cur, C 16 -SS-CS-mPEG, C 16 -CC-CS-mPEG, C ${ }_{16}$-SS-CSmPEG@Cur, and $\mathrm{C}_{16}-\mathrm{CC}-\mathrm{CS}-\mathrm{mPEG} @$ Cur for $24 \mathrm{~h}$ at a density of $1 \times 10^{5}$ cells. The cells cultured in plates were fixed with $3.7 \%$ formaldehyde and then permeabilized with $0.1 \%$ Triton X-100 in PBS. Then, the cells were incubated with TUNEL reaction mixture containing nucleotide mixture and terminal deoxynucleotidyl transferase (TdT) for $1 \mathrm{~h}$ and $1 \mathrm{mg} \mathrm{mL}-1$ of DAPI for $15 \mathrm{~min}$ at $37^{\circ} \mathrm{C}$, respectively. Stain cells were then rinsed with PBS and observed under a fluorescence microscope (Nikon Eclipse 80i).

\section{Apoptosis assay by FCM}

The MCF-7 cells $\left(5 \times 10^{5}\right.$ cells/well) in six-well culture plates were incubated with PBS, Cur, $\mathrm{C}_{16}-\mathrm{CC}-\mathrm{CS}-\mathrm{mPEG}$, $\mathrm{C}_{16}$-SS-CS-mPEG, $\mathrm{C}_{16}$-CC-CS-mPEG@Cur, and $\mathrm{C}_{16}$-SSCS-mPEG@Cur for 48 h, collected, and washed twice with PBS. To detect apoptosis, both adherent and floating cells were harvested together and resuspended in annexin $\mathrm{V}$ binding buffer (10 mM HEPES/NaOH, pH 7.4, $140 \mathrm{mM}$ $\mathrm{NaCl}, 2.5 \mathrm{mM} \mathrm{CaCl}_{2}$ ) at a concentration of $1 \times 10^{6}$ cells $/ \mathrm{mL}$. Subsequently, $5 \mathrm{~mL}$ of propidium iodide and $5 \mathrm{~mL}$ of 
FITC-conjugated annexin V were added to $100 \mathrm{~mL}$ of the cell suspension $\left(1 \times 10^{5}\right.$ cells $)$. The cells were incubated at room temperature for $15 \mathrm{~min}$ in the dark. Finally, $400 \mathrm{~mL}$ of annexin $\mathrm{V}$ binding buffer was added to each tube, and cells were analyzed by FCM (BD FACSAria).

\section{Quantitative real-time polymerase chain reaction (Q-PCR) and Western blotting analysis}

NIN/3T3 cells were precultured on six-well plates, at the density of $1 \times 10^{5}$ per well, and cultured at $37^{\circ} \mathrm{C}$ in $5 \% \mathrm{CO}_{2}$ atmosphere for $24 \mathrm{~h}$. Then, the cells were incubated with lipopolysaccharide (LPS), $\mathrm{C}_{16}$-CC-CS-mPEG, $\mathrm{C}_{16}$-SS-CS-mPEG, Cur, C ${ }_{16}$-CC-CS-mPEG@Cur, C ${ }_{16}$ SS-CS-mPEG@Cur, and PBS for 24 h. The total RNA was collected and isolated from the cells using a TRIzol Reagent (Invitrogen, Carlsbad, CA, USA). A total of $1 \mu \mathrm{g}$ of total RNA from each group was reverse transcribed into cDNA $(20 \mu \mathrm{L})$ using the PrimeScript First Strand cDNA synthesis kit. Thereafter, $2 \mu \mathrm{L}$ of cDNA was subjected to qPCR analysis using the SYBR Premix Ex TaqTM (TaKaRa Code: DRR041A).

$\mathrm{NIH} / 3 \mathrm{~T} 3$ cells $\left(1 \times 10^{5}\right.$ cells/well $)$ were seeded into 96-well plate and then cultured at $37^{\circ} \mathrm{C}$ for $24 \mathrm{~h}$. The cells were pretreated with Cur, $\mathrm{C}_{16}$-SS-CS-mPEG@Cur, and $\mathrm{C}_{16}$-CC-CS-mPEG@Cur for $4 \mathrm{~h}$ prior to LPS stimulation. After $48 \mathrm{~h}$ treatment with the delivery system, the cells were incubated with lysis buffer (Beyotime, Shanghai, People's Republic of China) to obtain total cellular proteins. The protein concentration in the lysate was determined using the bicinchoninic acid (BCA) assay. Samples $(20 \mu \mathrm{g})$ were separated by sodium dodecyl sulfate-polyacrylamide gel electrophoresis (SDS-PAGE) with 12\% acrylamide and transferred to a nitrocellulose membrane using a Western blot apparatus. Each membrane was blocked with 5\% bovine serum albumin for $2 \mathrm{~h}$, and then incubated overnight at $4^{\circ} \mathrm{C}$ with $1 \mu \mathrm{g} / \mathrm{mL}$ of a 1:2,000 dilution of primary antibody. HRP-conjugated tumor necrosis factor (TNF- $\alpha$; a 1:10,000 dilution) was used as the secondary antibody. Protein expression levels were determined by signal analysis using an image analyzer (Fuji-Film, Tokyo, Japan). $\beta$-Actin was used to confirm the comparable amount of proteins in each sample.

\section{Determination of the TNF- $\alpha$ secretion}

$\mathrm{NIH} / 3 \mathrm{~T} 3$ cells $\left(1 \times 10^{5}\right.$ cells/well) were seeded into 96-well plate and then cultured at $37^{\circ} \mathrm{C}$ for $24 \mathrm{~h}$. The cells were pretreated with Cur, $\mathrm{C}_{16}-\mathrm{SS}-\mathrm{CS}-\mathrm{mPEG} @ \mathrm{Cur}$, and $\mathrm{C}_{16}{ }^{-\mathrm{CC}}-\mathrm{CS}-$ mPEG@Cur for $4 \mathrm{~h}$ prior to LPS stimulation. LPS in medium was added at a final concentration of $1.0 \mathrm{mg} / \mathrm{mL}$. After incubating for $48 \mathrm{~h}$, the cell supernatants were collected, centrifuged at $800 \mathrm{rpm}$ for $5 \mathrm{~min}$, and the concentration of TNF- $\alpha$ was detected by enzyme-linked immunosorbent assay (ELISA) assay kit.

\section{Animals and tumor model}

Female nude C57/BL/6 mice (6 weeks old) were purchased from Sun Yat-sen University. The animals received care under pathogen-free conditions. Nude mice were kept under conditions controlled $50 \% \pm 5 \%$ of humidity and $25^{\circ} \mathrm{C} \pm 1^{\circ} \mathrm{C}$ of temperature and allowed free access to sterilized tap water and laboratory rodent chow. All the animal operations complied with the institutional and Chinese government guidelines for the care and use of experimental animals, and were approved by the Animal Care and Use Committee of Jinan University. To develop the tumor model, $1 \times 10^{6} \mathrm{MCF}-7$ cells suspended in $100 \mu \mathrm{L}$ of PBS were subcutaneously injected into the right breast of each mouse. The mice were used when tumor volumes reached $\sim 100 \mathrm{~mm}^{3}$.

\section{In vivo imaging}

For in vivo imaging, $200 \mu \mathrm{L}$ of saline (control), Cur, and $\mathrm{C}_{16}$-SS-CS-mPEG@Cur with a 0.54 mg/mL Cur equivalent concentration was intravenously injected into each mouse (doses: Cur mg/kg). In vivo fluorescence imaging was conducted using a Maestro in vivo optical imaging system (Cambridge Research and Instrumentation, Inc., Woburn, MA, USA). To detect Cur fluorescence, we used red light with the center wavelength at $661 \mathrm{~nm}$ as the excitation light and collected emission spectra from 600 to $850 \mathrm{~nm}$. Spectral unmixing was conducted by the Maestro software to remove the autofluorescence background.

\section{In vivo treatment}

The mice model was intravenously injected with $2.5 \mathrm{mg} / \mathrm{kg}$ $\mathrm{C}_{16}$-SS-CS-mPEG@Cur and saline (control) every day. The mice tumor sizes and body weights were recorded every 3 days; the tumor sizes were measured by Vernier caliper and calculated as length $\times$ width $\times$ height. Then, these mice were killed, and all the tumors were immediately harvested and weighed.

\section{Results}

\section{Characterization of $\mathrm{C}_{16}$-SS-CS-mPEG and $\mathrm{C}_{16}$-SS-CS-mPEG@Cur}

The synthesis procedure of $\mathrm{C}_{16}-\mathrm{SS}-\mathrm{COOH}$ or $\mathrm{C}_{16}-\mathrm{CC}-\mathrm{COOH}$ polymer is illustrated in Scheme 2. The synthesis of amphiphilic CS through the inclusion of $\mathrm{mPEG}, \mathrm{C}_{16}$-SS$\mathrm{COOH}$, or $\mathrm{C}_{16}-\mathrm{CC}-\mathrm{COOH}$ moieties using amine coupling 


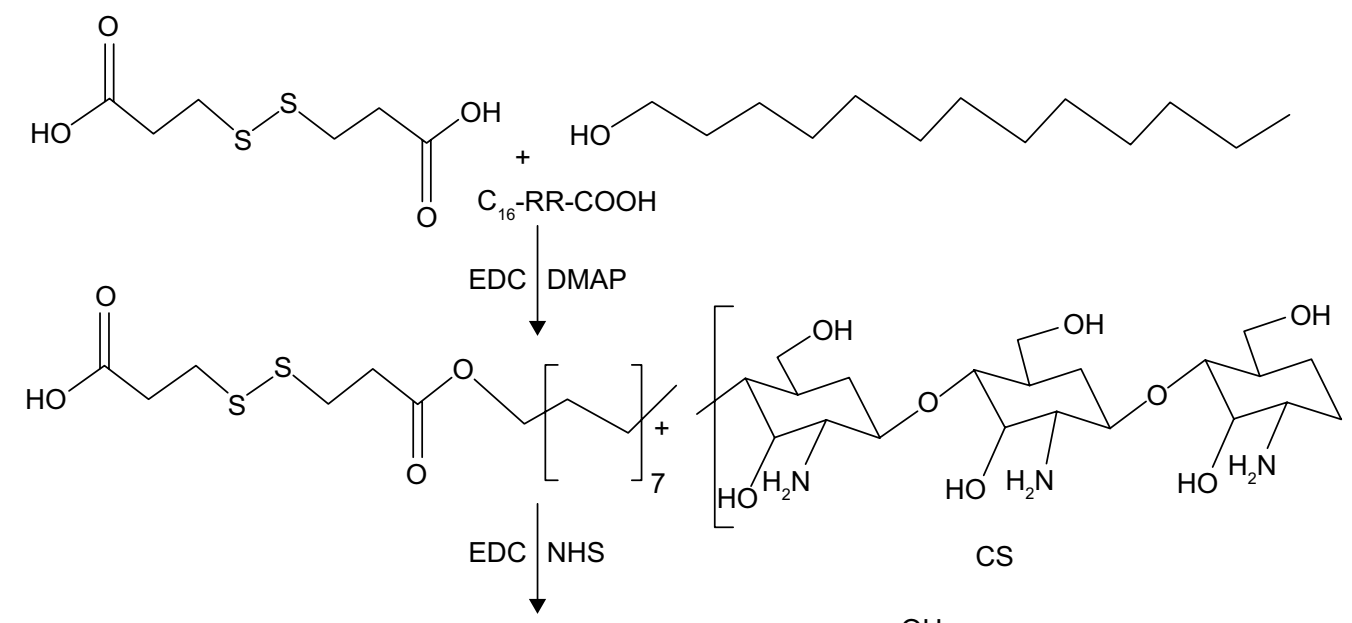

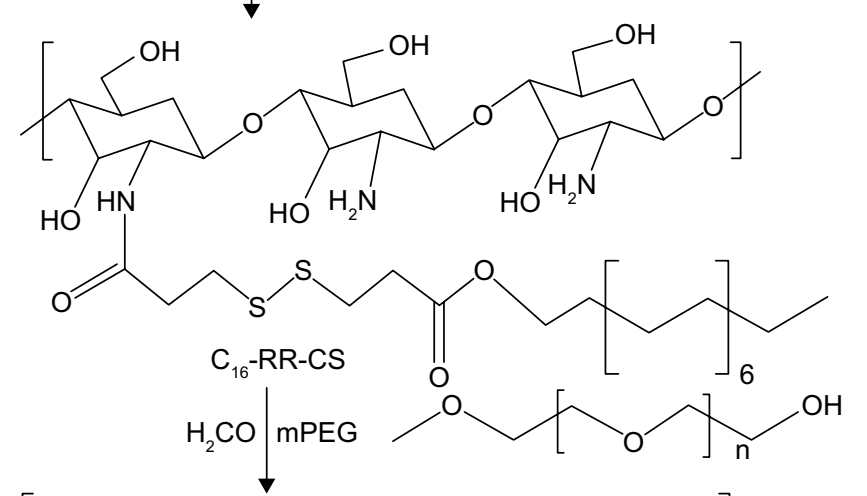

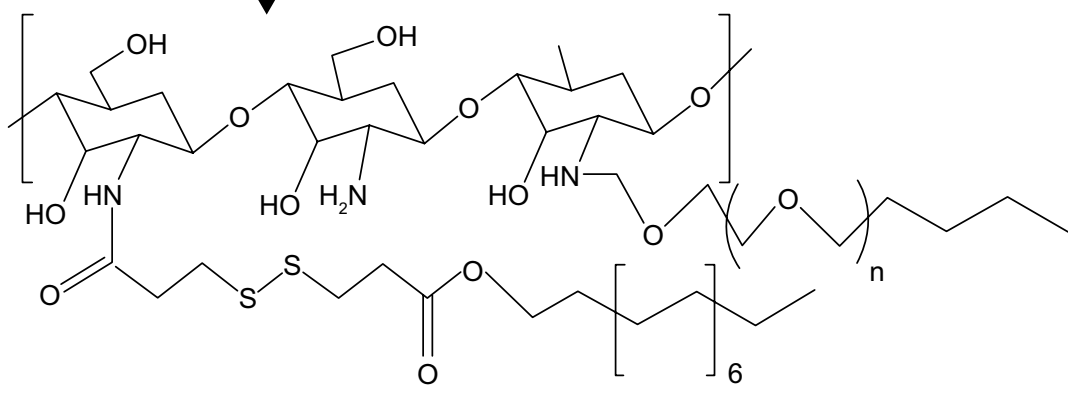

$\mathrm{C}_{16}$-RR-CS-mPEG

$-R R=-S S-\quad C_{16}-S S-C S-m P E G$

-CC- $\quad \mathrm{C}_{16}$-CC-CS-mPEG

Scheme 2 Synthesis pathway of the amphiphilic polymers.

Abbreviations: $C_{16}$-SS-CS-mPEG, monomethoxy-poly(ethylene glycol)-chitosan-S-S-hexadecyl; $C_{16}$-CC-CS-mPEG, monomethoxy-poly(ethylene glycol)-chitosan-C-Chexadecyl; Cur, curcumin; EDC, I-(3-dimethylaminopropyl)-3-ethylcarbodiimide; DMAP, 4-dimethylaminopyridine; NHS, N-hydroxysuccinimide.

chemistry was demonstrated by FTIR analysis. As shown in Figure 1A, peaks at $1,657 \mathrm{~cm}^{-1}$ and $1,600 \mathrm{~cm}^{-1}$ are the characteristic bands of CS. The slight increase in the amide I band at $\sim 1,650 \mathrm{~cm}^{-1}$ results from the amidation and simultaneous grafting of $\mathrm{C}_{16}-\mathrm{SS}-\mathrm{COOH}$ or $\mathrm{C}_{16}-\mathrm{CC}-\mathrm{COOH}$ into the CS. Furthermore, the slight increase in signal of methylene at $\sim 2,900 \mathrm{~cm}^{-1}$ was obtained from $\mathrm{mPEG}$ of $\mathrm{C}_{16}{ }^{-}$ SS-CS-mPEG or $\mathrm{C}_{16}$-SS-CS-mPEG, proving that a graft reaction between $\mathrm{CS}$ and $\mathrm{mPEG}$ occurred. Further characterization of the chemical composition of different compounds was performed by ${ }^{1} \mathrm{H}$ NMR analysis. Figure $1 \mathrm{~B}$ shows the
${ }^{1} \mathrm{H}$ NMR spectrum of $\mathrm{C}_{16}$-CC-CS-mPEG. A peak appeared at $2.0035 \mathrm{ppm}$, which indicated the acetyl group $\left(-\mathrm{NH}-\mathrm{O}-\mathrm{CH}_{3}\right)$ protons from the acetylated residues in CS. The peaks at 3.753 and $4.255 \mathrm{ppm}$ were assigned to $\mathrm{mPEG}$. Also, the structure of $\mathrm{C}_{16}$-SS-COOH was confirmed by ${ }^{1} \mathrm{H}$ NMR as shown in Figure S1; a peak appeared at 3.6876 ppm, which indicated the coupling of hexadecanol. The mPEG-CC-C 16 polymer was similarly prepared and used as a control.

In normal physiological aqueous environments, the conjugates spontaneously formed into micellar nanoparticles with hydrophobic and hydrophilic parts. ${ }^{18-20}$ As shown in 
A

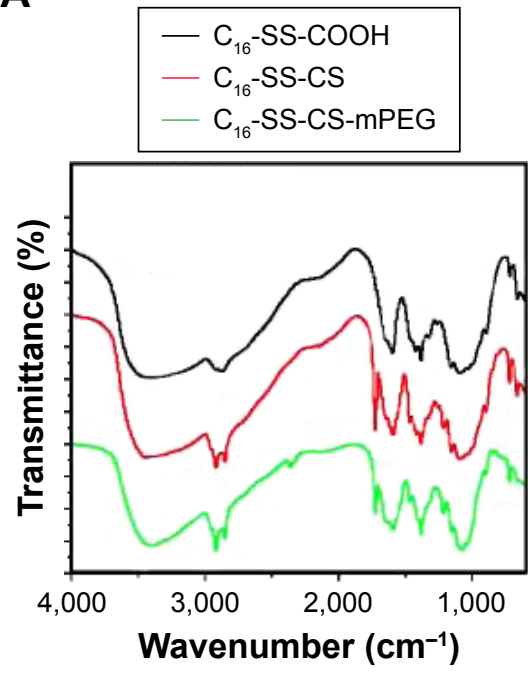

B

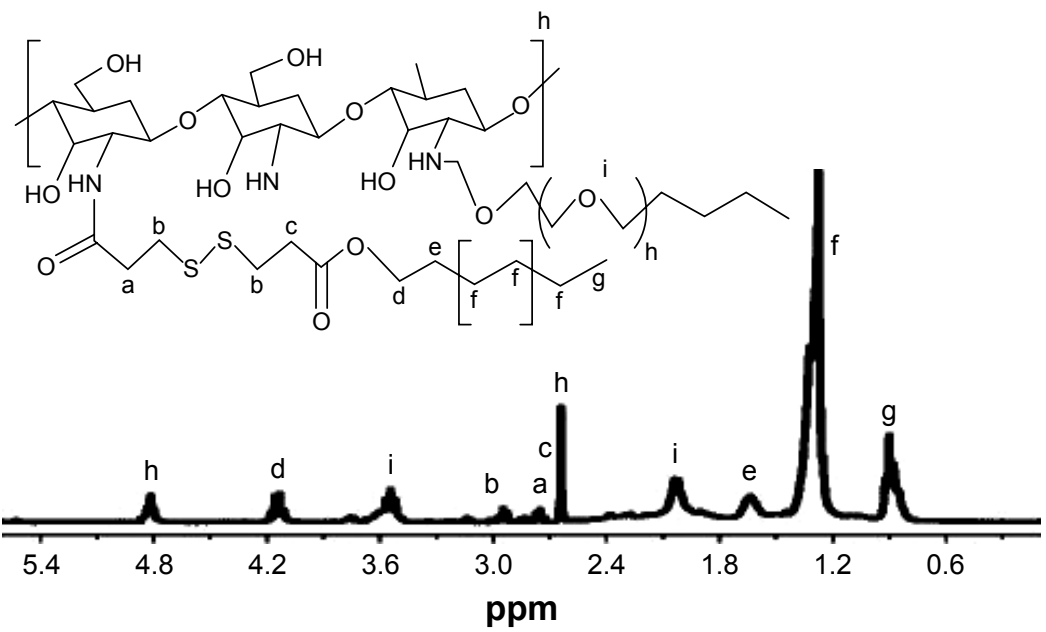

C

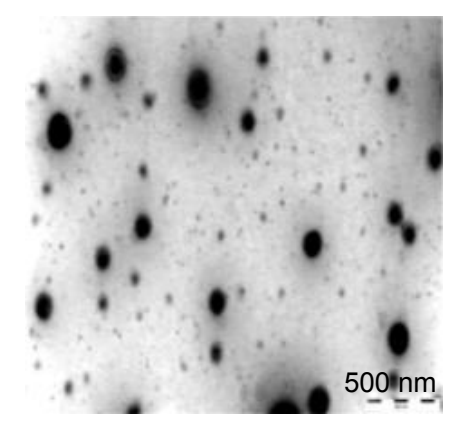

D

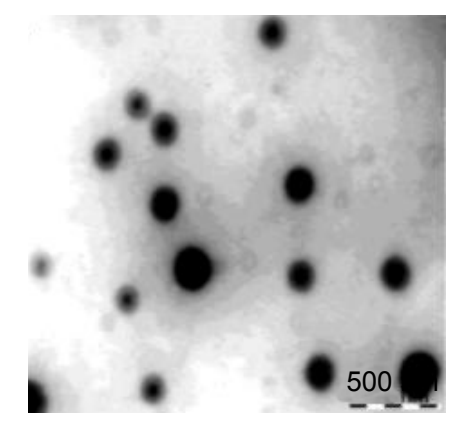

E
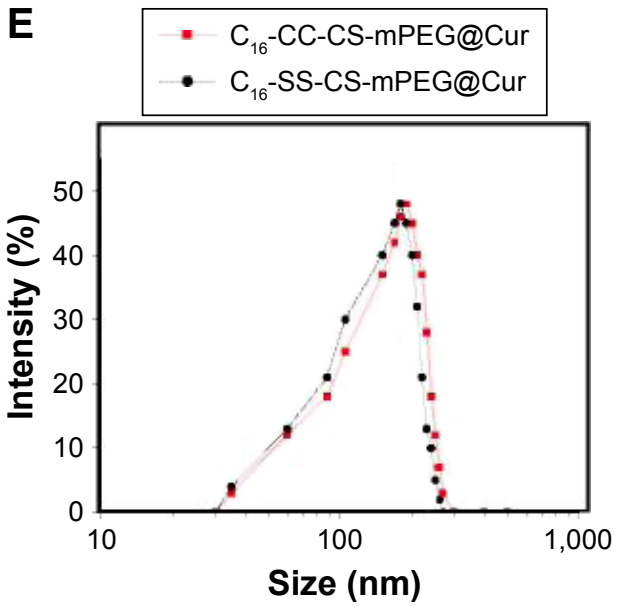

Figure I The characteristics of different micelles.

Notes: (A) FTIR spectra of $\mathrm{C}_{16}$-SS-COOH, $\mathrm{C}_{16}$-SS-CS, and $\mathrm{C}_{16}$-SS-CS-mPEG. (B) 'H NMR spectra of $\mathrm{C}_{16}$-SS-CS-mPEG polymer in D $\mathrm{O}$ (PPm). TEM micrographs of (C) C $\mathrm{C}_{16}$-SSCS-mPEG@Cur micelles and (D) $C_{16}$-CC-CS-mPEG@Cur micelles. The size distribution by DLS of (E) $\mathrm{C}_{16}$-SS-CS-mPEG@Cur micelles and $\mathrm{C}_{16}$-CC-CS-mPEG@Cur micelles.

Abbreviations: FTIR, Fourier transform-infrared spectrometry; $\mathrm{C}_{16}$-SS-CS-mPEG, monomethoxy-poly(ethylene glycol)-chitosan-S-S-hexadecyl; $\mathrm{C}_{16}$-CC-CS-mPEG, monomethoxy-poly(ethylene glycol)-chitosan-C-C-hexadecyl; Cur, curcumin; 'H NMR, 'H nuclear magnetic resonance; TEM, transmission electron microscopy.

Figure 1C and D, the $\mathrm{C}_{16}-\mathrm{SS}-\mathrm{CS}-\mathrm{mPEG} @ \mathrm{Cur}$ and $\mathrm{C}_{16}-\mathrm{CC}$ CS-mPEG@Cur micelles were uniform and spherical with diameters between $95-121 \mathrm{~nm}$ and $87-155 \mathrm{~nm}$ as observed from TEM measurements, respectively. The hydrodynamic diameters of $\mathrm{C}_{16}$-SS-CS-mPEG@Cur and $\mathrm{C}_{16}$-CC-CSmPEG@Cur micelles determined by dynamic light scattering (DLS) were $173 \pm 14$ and $188 \pm 22 \mathrm{~nm}$, respectively (Figure 1E). The average diameters were slightly larger than those found in the TEM results because of the different detection conditions used. TEM images are captured when nanoparticles are in a dried state, whereas DLS gives the hydrodynamic size thereof. Similar results arose elsewhere. ${ }^{21}$ These results indicated that both $\mathrm{C}_{16}$-SS-CSmPEG@Cur and 16 $_{16}$ CC-CS-mPEG@Cur formulations self-assembled into micelles with sizes suitable for passive intratumoral targeting accumulation of antineoplastic drug through the EPR effect. ${ }^{22,23}$

\section{In vitro drug release from micelles}

Disulfide linkages can be readily reduced into free thiols in the presence of reducing agents. The disulfide bridge linkage between $\mathrm{CS}$ and $\mathrm{C}_{16}$ makes $\mathrm{C}_{16}$-SS-CS-mPEG micelles reductively breakable in response to DTT, which leads to drug release. The calibration curves of Cur are shown in Figure $2 \mathrm{~A}$, and Cur load capacity of $\mathrm{C}_{16}$-SS-CSmPEG and $\mathrm{C}_{16}-\mathrm{CC}-\mathrm{CS}-\mathrm{mPEG}$ was 7.62 and $8.21 \mathrm{wt} \%$, respectively. The in vitro release behaviors of Cur from $\mathrm{C}_{16}$-SS-CS-mPEG@Cur were detected in water at different concentrations of DTT; $\mathrm{C}_{16}-\mathrm{CC}-\mathrm{CS}-\mathrm{mPEG} @$ Cur served as a control. 

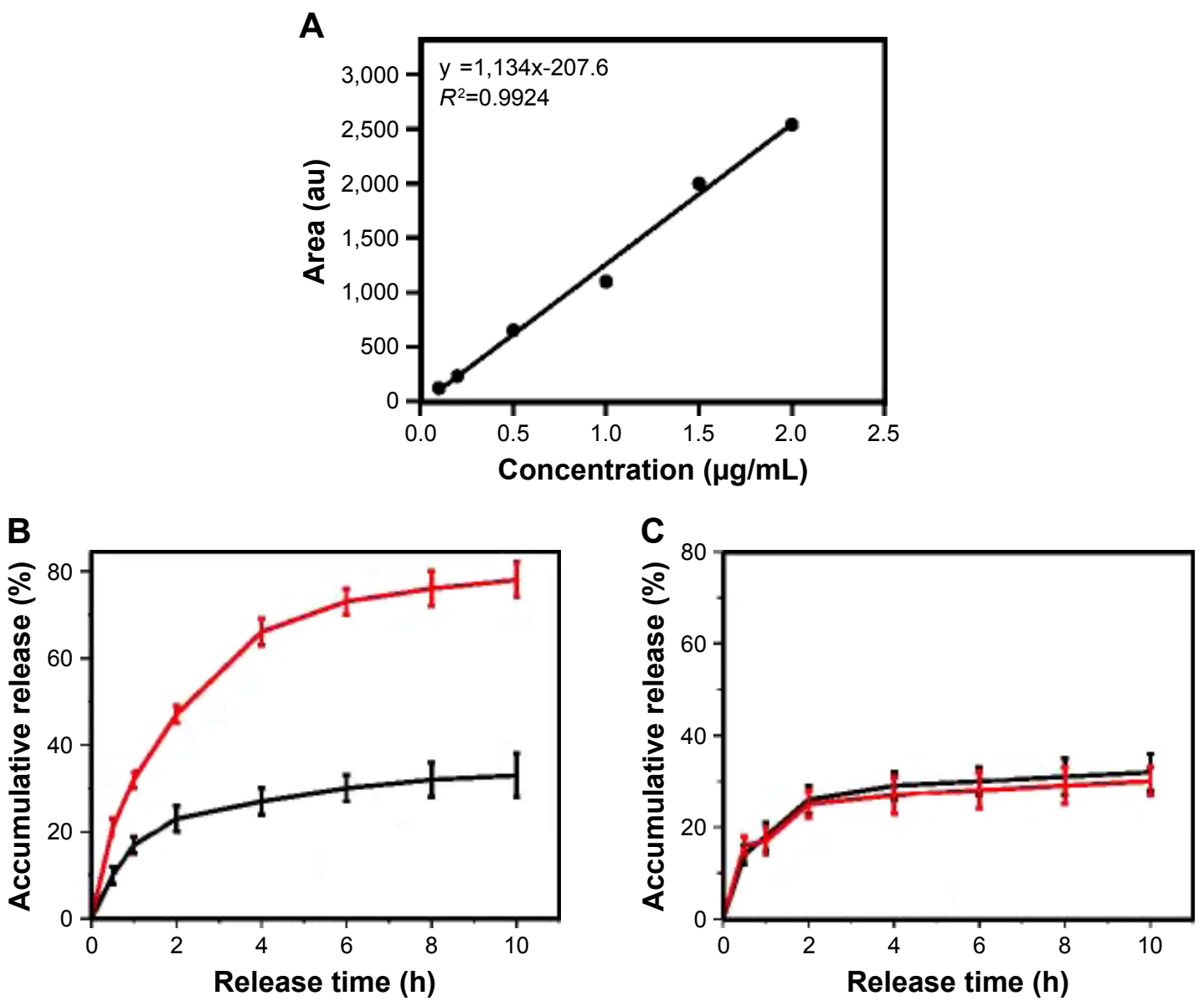

PBS $10 \mathrm{mM} \mathrm{DTT}$

Figure 2 In vitro drug release from micelles.

Notes: (A) Calibration curves of Cur at concentrations of 0.1-2.0 $\mu \mathrm{g} \mathrm{mL} \mathrm{L}^{-1}$. Reduction-triggered release of Cur from (B) $\mathrm{C}_{16}$-SS-CS-mPEG@Cur micelles and (C) Cur-loaded $\mathrm{C}_{16}$-CC-CS-mPEG@Cur micelles in PBS (10 mM, pH 7.4) with, or without, 10 mM DTT. Data are shown as mean \pm SD ( $\left.n=3\right)$.

Abbreviations: $C_{16}$-SS-CS-mPEG, monomethoxy-poly(ethylene glycol)-chitosan-S-S-hexadecyl; $C_{16}$-CC-CS-mPEG, monomethoxy-poly(ethylene glycol)-chitosan-C-Chexadecyl; Cur, curcumin; PBS, phosphate-buffered saline; SD, standard deviation.

As shown in Figure $2 \mathrm{~B}$ and $\mathrm{C}$, the release profiles showed a burst release at the early stage for Cur from $\mathrm{C}_{16}$-SS-CS-mPEG@Cur, 87.4\% of Cur was released in the presence of $10 \mathrm{mM}$ DTT in $10 \mathrm{~h}$. However, there was only $25.9 \%$ Cur released from $\mathrm{C}_{16}$-SS-CS-mPEG@Cur after $10 \mathrm{~h}$ in the absence of DTT. In contrast, only $27.6 \%$ and $28.3 \%$ Cur was released from $\mathrm{C}_{16}$-CC-CS-mPEG@Cur with or without DTT, respectively, indicating that the addition of DTT had a negligible effect on the release of Cur from the $\mathrm{C}_{16}$-CC-CS-mPEG@Cur micelles. These results could have arisen because of the cleavage of the disulfide bond in the $\mathrm{C}_{16}$-SS-CS-mPEG@Cur polymer caused by DTT, which broke the structure of the micellar form. These results suggested that $\mathrm{C}_{16}$-SS-CS-mPEG@Cur micelles may achieve controlled-release drug effects in the presence of reducing agents.

\section{Endocytosis inhibition}

As a key factor for drug delivery nanoparticle systems, the endocytosis pathway of $\mathrm{C}_{16}$-SS-CS-mPEG@Cur and $\mathrm{C}_{16}$-CC-CS-mPEG@Cur micelles must be determined. There are three main endocytic pathways for nanoparticles: macropinocytosis, clathrin-mediated endocytosis, and caveolae-mediated endocytosis. ${ }^{24}$ In this assay, both $\mathrm{C}_{16}$-SSCS-mPEG@Cur and $\mathrm{C}_{16}$-CC-CS-mPEG@Cur micelles were analyzed using CLSM and FCM. Three different types of endocytosis inhibitors were used to inhibit different endocytosis routes: we used $10 \mathrm{mM}$ MBCD to inhibit caveolaemediated endocytosis, $0.5 \mathrm{M}$ hypertonic sucrose to inhibit clathrin-mediated endocytosis, and $10 \mathrm{mM}$ cytochalasin D to inhibit macropinocytosis.

Figure 3A shows CLSM images of Cur fluorescence in MCF-7 cells after incubation with $\mathrm{C}_{16}$-SS-CS-mPEG@Cur 
A

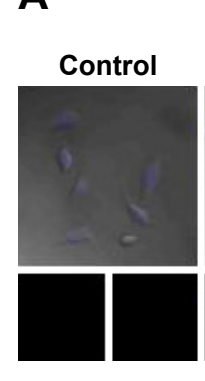

C mPEG@Cur
Sucrose
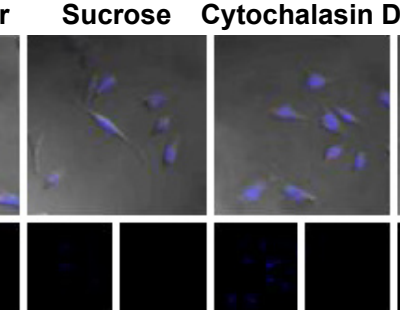

$\mathrm{C}_{16}$-CC-CS-
mPEG@Cur

MBCD
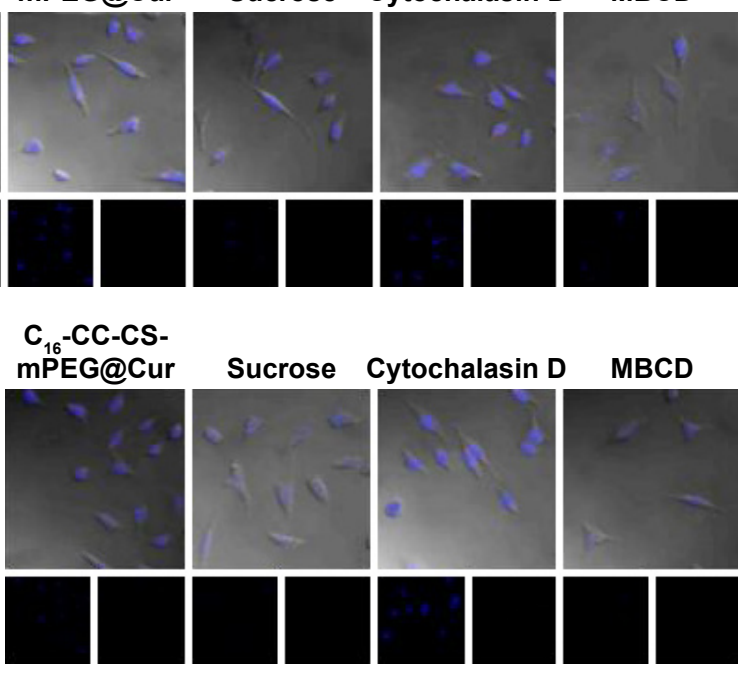

B

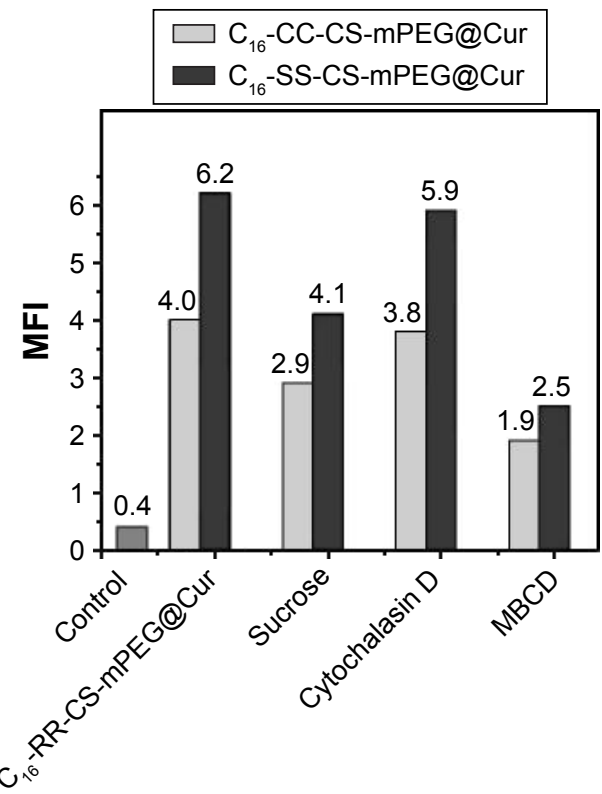

Figure 3 Effect of endocytosis inhibitors on the uptake of different micelles.

Note: Effect of endocytosis inhibitors on the uptake of mPEG-S-S-C 16 @Cur micelles and mPEG-C-C-C 16 @Cur micelles on MCF-7 cells using CLSM (A) and FCM (B) analyses.

Abbreviations: $\mathrm{C}_{16}$-SS-CS-mPEG, monomethoxy-poly(ethylene glycol)-chitosan-S-S-hexadecyl; $\mathrm{C}_{16}$-CC-CS-mPEG, monomethoxy-poly(ethylene glycol)-chitosan-C-C-hexadecyl; Cur, curcumin; CLSM, confocal laser scanning microscope; FCM, flow cytometry; MFI, mean fluorescence intensity; MBCD, methyl- $\beta$-cyclodextrin.

and $\mathrm{C}_{16}$-CC-CS-mPEG@Cur micelles with, or without, endocytosis inhibitors for 2 h. For $\mathrm{C}_{16}$-SS-CS-mPEG@Cur micelles, the Cur fluorescence of cells treated with MBCD and hypertonic sucrose was weaker than that of cells incubated without an inhibitor: this was especially obvious in cells cultured with hypertonic sucrose. Cells cultured with cytochalasin D did not influence Cur fluorescence intensity compared with that in cells incubated without an inhibitor. The same result was obtained from the $\mathrm{C}_{16}$-CC-CS-mPEG@Cur micelle treatment group. The Cur fluorescence intensity represents the amount of cellular uptake of micelles. Meanwhile, the results from FCM showed the same behavior. As shown in Figure 3B, when treated with $\mathrm{MBCD}$, the cellular uptake efficiencies of $\mathrm{C}_{16}$-SS-CS-mPEG@Cur and $\mathrm{C}_{16}-\mathrm{CC}$ CS-mPEG@Cur micelles decreased to 70.3\% and 68.6\%, respectively. However, after treatment with hypertonic sucrose, the cellular uptake of $\mathrm{C}_{16}$-SS-CS-mPEG@Cur and $\mathrm{C}_{16}$-CC-CS-mPEG@Cur micelles significantly decreased to $43.8 \%$ and $44.2 \%$, respectively. The inhibition with hypertonic sucrose and MBCD indicated that the pathways for micelle cellular uptake were caveolae-mediated endocytosis and clathrin-mediated endocytosis with the principal pathway being clathrin-mediated endocytosis. There was no obvious difference in uptake pathway between $\mathrm{C}_{16}-\mathrm{SS}$ CS-mPEG@Cur and $\mathrm{C}_{16}$-CC-CS-mPEG@Cur micelles or even in cellular uptake efficiency. Therefore, both cellular uptake pathway and cellular uptake efficiency will not affect the following assays.

\section{Intracellular drug release}

Since the internalization of both micelles $\left(\mathrm{C}_{16}-\mathrm{SS}-\mathrm{CS}\right.$ mPEG@Cur and $\mathrm{C}_{16}$-CC-CS-mPEG@Cur) was mediated by the same endocytosis pathway, mainly by clathrin-mediated endocytosis, intracellular drug release of both micelles should be investigated. CLSM was used to observe MCF-7 cells after incubation with Cur-loaded micelles and free Cur for different times. As shown in Figure 4A and B, compared with free Cur treatment, cells treated with both $\mathrm{C}_{16}-\mathrm{SS}-\mathrm{CS}$ mPEG@Cur and $\mathrm{C}_{16}$-CC-CS-mPEG@Cur showed stronger fluorescence in the cytoplasm after $12 \mathrm{~h}$. This may have been because of the drug delivery system enhancing the water solubility of Cur, which led to higher cellular uptake. ${ }^{25}$ Meanwhile, there was a significant difference between

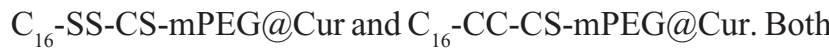
micelles could be accumulated by Cur rapidly in the cells in $2 \mathrm{~h}$ and reached their maximum concentration in $12 \mathrm{~h}$. However, when treated with $\mathrm{C}_{16}$-SS-CS-mPEG@Cur they still showed strong fluorescence while fluorescence showed visible decreases when treated with $\mathrm{C}_{16}$-CC-CS-mPEG@Cur after $24 \mathrm{~h}$, even remaining obvious after $48 \mathrm{~h}$. These results may have been due to unreleased Cur in $\mathrm{C}_{16}-\mathrm{CC}-\mathrm{CS}$ mPEG@Cur being excreted with micelles, in contrast Cur 
A
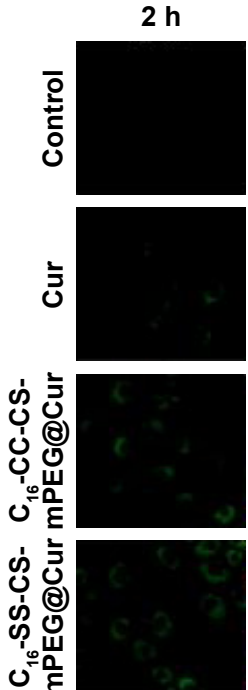

$12 \mathrm{~h}$
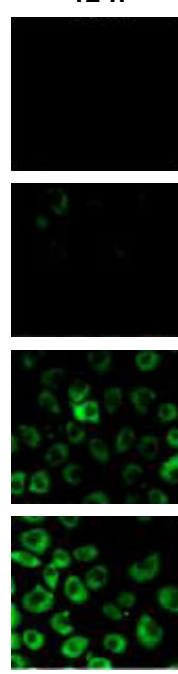

$24 \mathrm{~h}$
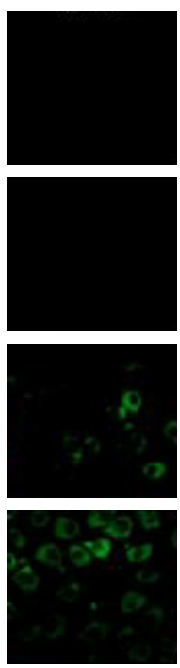

$48 \mathrm{~h}$
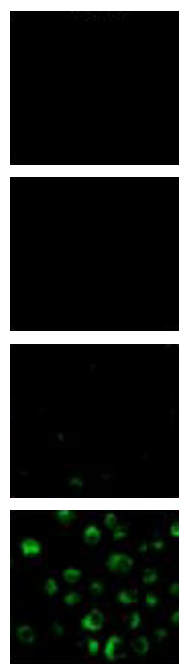

B
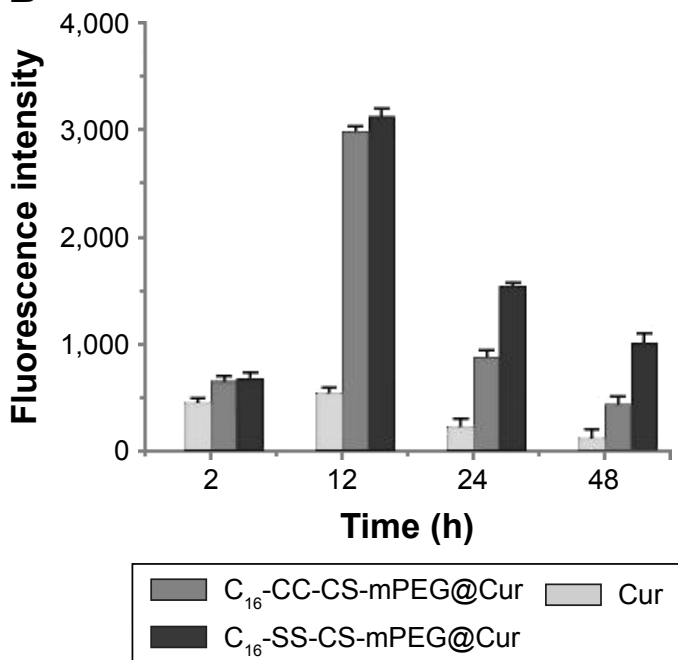

Figure 4 (A and B) CLSM images of MCF-7 cells after treatment with PBS, Cur, C 16 -CC-CS-mPEG@Cur, and C 16 -SS-CS-mPEG@Cur for 2, I2, 24, and 48 h and corresponding quantitative analysis of Cur fluorescence intensity.

Note: The control exhibited no fluorescence.

Abbreviations: CLSM, confocal laser scanning microscope; PBS, phosphate-buffered saline; $\mathrm{C}_{16}$-SS-CS-mPEG, monomethoxy-poly(ethylene glycol)-chitosan-S-S-hexadecyl; $\mathrm{C}_{16}$-CC-CS-mPEG, monomethoxy-poly(ethylene glycol)-chitosan-C-C-hexadecyl; Cur, curcumin.

released from $\mathrm{C}_{16}$-SS-CS-mPEG@Cur remained in the cells and played a pharmacodynamic role. Combined with the results of the drugs release assay (Figure 2), it was assumed that the $\mathrm{C}_{16}$-SS-CS-mPEG micelle structure would degrade when exposed to a reducing agent such as GSH in cells, and thus release drugs therein. Therefore, this $\mathrm{C}_{16}$-SS-CS-mPEG micelle was sensitive to reducing agents and could be a suitable redox-responsive drug carrier.

\section{In vitro cytotoxicity assay}

In this work, an MTT assay was conducted to compare the toxicities of different compounds against MCF-7 cells and HEK-293 cells. As shown in Figure 5A, compared with the control, HEK-293 cells were not influenced when incubated with both blank micelles, which indicated the noncytotoxicity and good biocompatibility of the two micelles, which was an essential property of such drug carriers.

To investigate the cytotoxic effects, MCF-7 cells were incubated with different compounds for $24 \mathrm{~h}$, followed by quantification of cell viability. Figure 5B shows that free Cur and drug-loaded micelles led to decreased cell viability after incubation. In detail, the cell survival rate was reduced to $72.2 \%$ after treatment with free Cur, while the value was $13.4 \%$ with the use of $\mathrm{C}_{16}$-SS-CS-mPEG@Cur. In addition, the cytotoxicity of $\mathrm{C}_{16}$-CC-CS-mPEG@Cur was slightly higher than that of the free Cur group with the cell survival rate of $63.3 \%$. This could have been due to enhanced cellular uptake of drugs and the much faster release thereof from $\mathrm{C}_{16}-\mathrm{SS}$ CS-mPEG micelles by cleavage of the disulfide bond, which was ensured by intracellular drug release assay (Figure 4). Consequently, the greater the dose released in cells, the higher the antitumor efficiency. The cell apoptosis was detected by annexin V/propidium iodide (PI) double staining and TUNEL assay and DAPI staining. As shown in Figure $5 \mathrm{C}$ and D, the cells incubated with the $\mathrm{C}_{16}$-SS-CS-mPEG@Cur cause obvious cells apoptosis than other nanoparticles. These results indicated that the drug delivery of redox-responsive $\mathrm{C}_{16}$-SS-CS-mPEG@ Cur systems had potential for chemotherapy and that it may have better tumor inhibition capability in vivo.

\section{Anti-inflammation effects}

The inflammatory microenvironment around a tumor plays an important role in tumor formation, growth, and metastasis. ${ }^{26}$ In this work, one of the purposes of delivering Cur was to exploit its anti-inflammatory effect on the area around a tumor to achieve a combination therapy effect. Inflammatory cell models were established by NIH 3T3 cells stimulated with LPS. ${ }^{27}$ The cellular uptake of Cur was assessed by FACS analysis. Figure 6A shows that there was no significant difference in intracellular cumulative amount of Cur after treatment with $\mathrm{C}_{16}$-CC-CS-mPEG@Cur and $\mathrm{C}_{16}$-SS-CSmPEG@Cur for 24 h, but there was about three times more than that found after treatment with free Cur, which indicated that drug-loaded micelles significantly enhanced the inflammatory cellular uptake of Cur.

To test the anti-inflammatory effects of drug-loaded micelles, we investigated, in vitro, the effect of different compounds on the expression of TNF- $\alpha$. TNF- $\alpha$ is a 


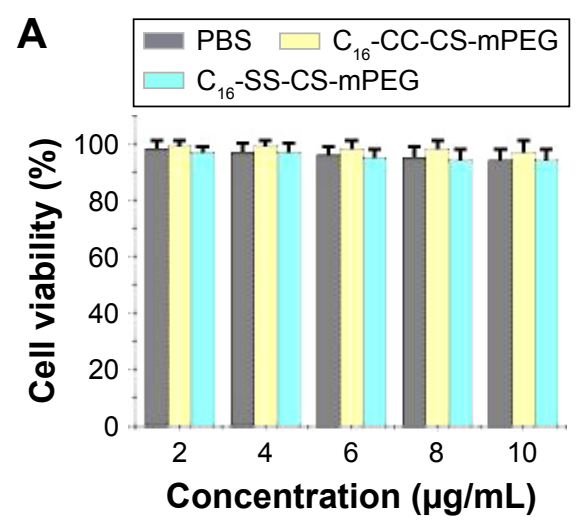

D
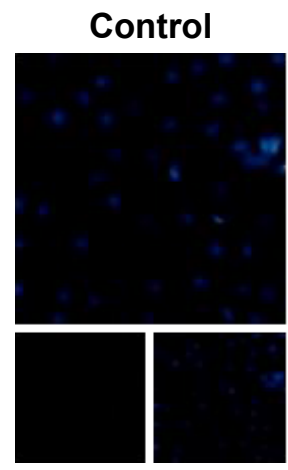
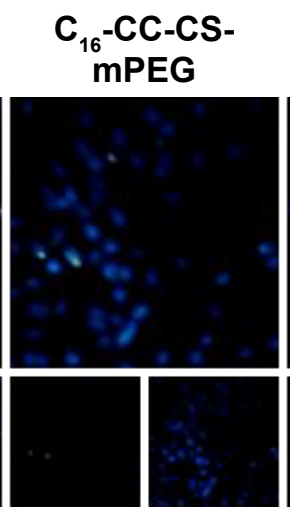

$B$

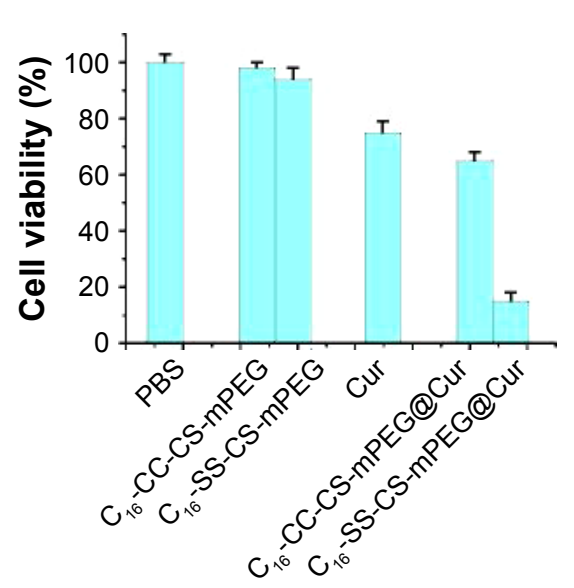

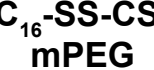

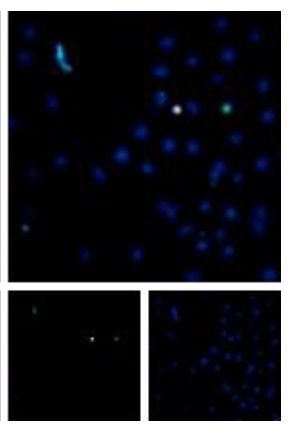

C

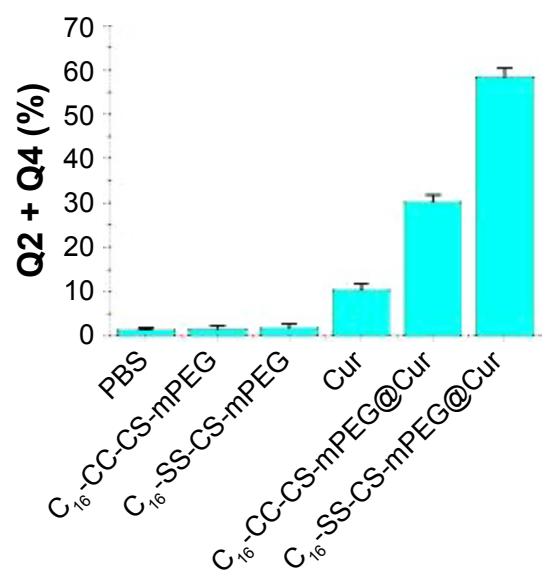

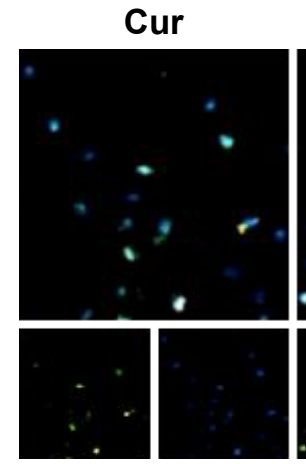

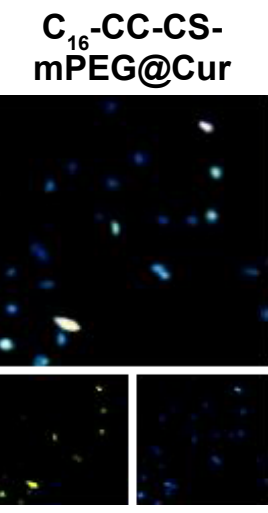

$\mathrm{C}_{16}$-SS-CSmPEG@Cur

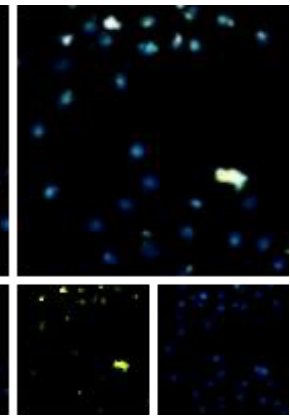

Figure 5 Cell viability and induction of apoptosis in MCF-7 cells.

Notes: (A) MTT assay of $C_{16}$-SS-CS-mPEG micelles and $C_{16}$-CC-CS-mPEG micelles in HEK293 cells after incubation for 24 h. (B) Cytotoxicity effects on MCF-7 cells of different micelles with, or without, drugs being loaded after incubation for 24 h. Measurement of cell apoptosis by (C) annexin V/PI double staining and (D) TUNEL assay and DAPI staining.

Abbreviations: $\mathrm{C}_{16}$-SS-CS-mPEG, monomethoxy-poly(ethylene glycol)-chitosan-S-S-hexadecyl; $\mathrm{C}_{16}$-CC-CS-mPEG, monomethoxy-poly(ethylene glycol)-chitosan-C-C-hexadecyl; Cur, curcumin; PBS, phosphate-buffered saline.

pro-inflammatory cytokine, which induces some proinflammatory cytokines including IL-1 $\beta$, IL-6, IL-8, and itself by activation of NF-KB or MAPKs, and these cytokines play important roles in various inflammatory diseases. ${ }^{28,29}$ Decreased TNF- $\alpha$ expression can be used to measure the anti-inflammatory efficiency. The expression of TNF- $\alpha$ was determined by real-time quantification PCR (Q-PCR) analysis and Western blotting assay. As shown in Figure 6B, the mRNA expressions of TNF- $\alpha$ increase significantly after stimulation with LPS and then decrease gradually within $\mathrm{C}_{16}$-SS-CS-mPEG@Cur. As shown in Figure 6C, NIH 3 T3 cells stimulated with LPS significantly enhanced the expression of TNF- $\alpha$, whereas treatment with $\mathrm{C}_{16}-\mathrm{SS}$ CS-mPEG@Cur significantly suppressed the expression of TNF- $\alpha$ in LPS-stimulated cells. Cur and $\mathrm{C}_{16}-\mathrm{CC}-\mathrm{CS}$ mPEG@Cur micelles slightly decreased TNF- $\alpha$ expression. These results could have been due to Cur-loaded $\mathrm{C}_{16}-\mathrm{SS}$ CS-mPEG micelles being able to release Cur to cytoplasmic effect rapidly allowing it to play its anti-inflammatory pharmacological roles. In contrast, Cur encapsulated in $\mathrm{C}_{16}$-CC-CS-mPEG@Cur could not be released to exert its anti-inflammatory effects. This result was consistent with results of the previous intracellular drug release assay (Figure 4). Quantitative data from the ELISA experiment (Figure 6D) showed that, after stimulation with LPS, the concentration of TNF- $\alpha$ rose to $\sim 34.8 \mathrm{pg} / \mathrm{mL}$. A total of 37.5 and $6.8 \mathrm{pg} / \mathrm{mL}$ of TNF- $\alpha$ were treated with $\mathrm{C}_{16}$-CC-CSmPEG@Cur and $\mathrm{C}_{16}$-SS-CS-mPEG@Cur, respectively. This result confirmed the result of the previous Western blotting assay. Taken together, Cur could be released from $\mathrm{C}_{16}-\mathrm{SS}-$ CS-mPEG@Cur rapidly to play its anti-inflammatory role.

\section{In vivo fluorescence imaging and biodistribution}

As an effective delivery system for anticancer agents, a potential carrier should be able to deliver the drugs directly 
A

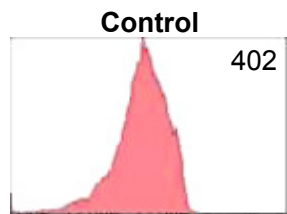

$\mathrm{C}_{16}$-CC-CS-

mPEG@Cur

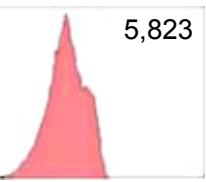

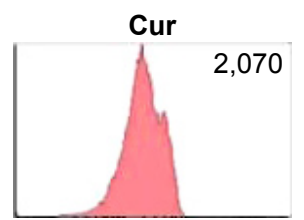

$\mathrm{C}_{16}$-SS-CS-

mPEG@Cur

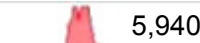

\section{C}
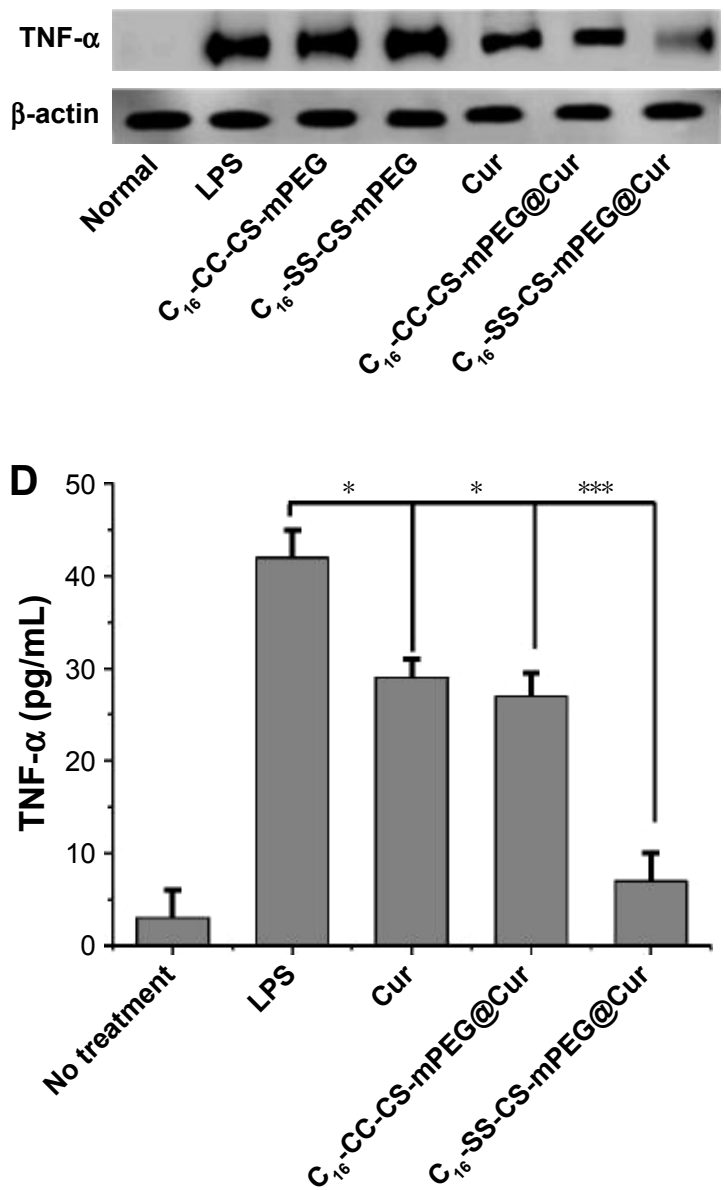

Figure 6 Anti-inflammatory effect in vitro.

Notes: (A) Histograms of Cur uptake in NIH/3T3 LPS-stimulated cells after 12 h incubation of Cur, CI6-SS-CS-mPEG@Cur, and CI6-CC-CS-mPEG@Cur. (B) Q-PCR analysis was used to evaluate mRNA levels. Anti-inflammatory effect of Cur, CI6-SS-CS-mPEG@Cur, and CI6-CC-CS-mPEG@Cur on TNF- $\alpha$ inflammatory mediator release in NIH/3T3 LPS-stimulated cells analyzed by $(\mathbf{C})$ Western blot experiment and (D) enzyme-linked immunosorbent assay experiment. Data are shown as mean \pm SD $(\mathrm{n}=3) . * \mathrm{p}<0.05, * * * \mathrm{p}<0.001$.

Abbreviations: $\mathrm{C}_{16}$-SS-CS-mPEG, monomethoxy-poly(ethylene glycol)-chitosan-S-S-hexadecyl; $\mathrm{C}_{16}$-CC-CS-mPEG, monomethoxy-poly(ethylene glycol)-chitosan-C-Chexadecyl; Cur, curcumin; LPS, lipopolysaccharide; Q-PCR, quantitative real-time polymerase chain reaction; TNF- $\alpha$, tumor necrosis factor $\alpha$; SD, standard deviation.

into tumor tissues to achieve tumor-targeted therapy. To evaluate whether $\mathrm{C}_{16}$-SS-CS-mPEG@Cur could efficiently assist in Cur accumulation in tumors, the fluorescence of Cur was observed using an IVIS Lumina imaging system at $4 \mathrm{~h}$ postadministration. Figure 7A shows the in vivo images after intravenous injection of free Cur and $\mathrm{C}_{16}$-SS-CS-mPEG@Cur into MCF-7 tumor-bearing nude mice. The fluorescence of tumor tissue, and other major organs of mice, is shown in Figure 7B. Through system modulation, there were no signals in the tumor tissues in the blank group (injected with saline), suggesting no autofluorescence interfered with the in vivo images, and the fluorescence signal intensity of in vivo imaging was close to a true reflection of Cur concentration. There was a significant difference in biodistribution of $\mathrm{Cur}$ between free Cur and $\mathrm{C}_{16}-\mathrm{SS}-\mathrm{CS}-\mathrm{mPEG} @$ Cur treatments. The $\mathrm{C}_{16}-\mathrm{SS}-$ CS-mPEG@Cur group showed strong fluorescence in the tumor region and was almost twice as strong as that in the free Cur group. Moreover, the fluorescence clearly accumulated in both the liver and kidney after treatment with free Cur; in contrast, there was only slight fluorescence accumulated in those regions. This increase in tumor-targeting efficiency provided by nanoparticle delivery might be due to an EPR effect and the controlled release property of $\mathrm{C}_{16}-\mathrm{SS}$ CS-mPEG micelles. The results indicated that $\mathrm{C}_{16}-\mathrm{SS}-\mathrm{CS}-$ mPEG@Cur has enhanced tumor-targeted delivery, which leads to enhance Cur accumulation in tumor.

\section{Antitumor effects in vivo}

To investigate the therapeutic effect of $\mathrm{C}_{16}$-SS-CSmPEG@Cur on tumors in vivo, a breast tumor xenograft model was established by subcutaneous injection of MCF-7 cells of nude mice (five per group). Treatments started when 
A

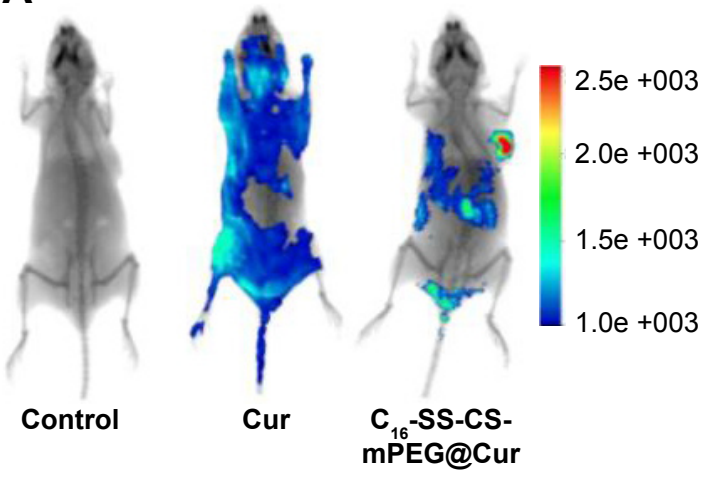

B

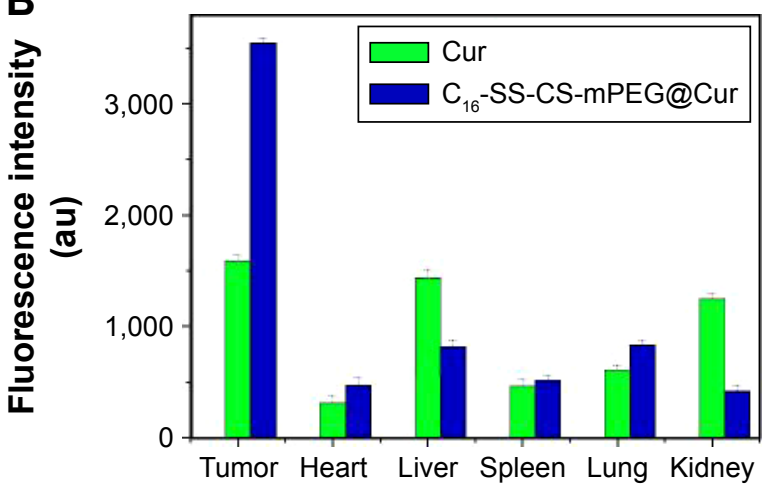

Figure 7 In vivo fluorescence imaging and fluorescence intensity of tissues.

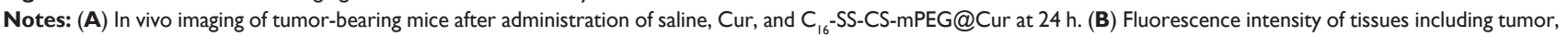
heart, liver, spleen, lung, and kidney removed from tumor-bearing nude mice after sacrificing the mice at 24 h postinjection of saline, Cur, and $\mathrm{C}_{16}{ }^{-S S}$-CS-mPEG@Cur. The saline exhibited no fluorescence.

Abbreviations: $\mathrm{C}_{16}$-SS-CS-mPEG, monomethoxy-poly(ethylene glycol)-chitosan-S-S-hexadecyl; Cur, curcumin.

the tumor volume reached $\sim 100 \mathrm{~mm}^{3}$. Injections occurred for a total of seven times on alternate days, and both mouse body weights and tumor size were recorded simultaneously. The average volume of the tumor was monitored every 3 days. As shown in Figure 8A, evidence was provided of the therapeutic effect of $\mathrm{C}_{16}$-SS-CS-mPEG@Cur micelles. Tumors treated with saline and $\mathrm{C}_{16}-\mathrm{SS}-\mathrm{CS}-\mathrm{mPEG}$ showed continuous growth with similar tendencies at a relatively high rate with a final tumor volume of $\sim 1,300 \mathrm{~mm}^{3}$, suggesting that the MCF-7 tumor growth was unaffected by saline and non-drug-loaded micelles. Free Cur groups showed slight inhibition. Most importantly, the $\mathrm{C}_{16}$-SS-CS-mPEG@Curtreated group exhibited the highest inhibition of those tested, with a final tumor volume of $\sim 205 \mathrm{~mm}^{3}$. Compared with the MTT assay (Figure 5), this result may have been due to passive targeting and the controlled release properties of $\mathrm{C}_{16}$-SS-CS-mPEG@Cur micelles.

After 30 days' treatment, the mice were sacrificed, and the tumor masses were measured. Figure $8 \mathrm{C}$ shows the solid tumors from each group: intuitive, and similar, results were obtained among which, the $\mathrm{C}_{16}$-SS-CS-mPEG@Cur group exhibited the most dramatic inhibition of tumor growth. Figure 8D further reveals the inhibitory effect based on average tumor mass in each group. While the average tumor mass in the saline group was $0.635 \pm 0.027 \mathrm{~g}$, that of the free Cur and $\mathrm{C}_{16}$-SS-CS-mPEG@Cur groups reached 0.337 $\pm 0.045 \mathrm{~g}$ and $0.089 \pm 0.072 \mathrm{~g}$, respectively. Now, tumor growth inhibition reached $47.2 \%$ and $85.9 \%$, respectively. These results demonstrated that $\mathrm{C}_{16}$-SS-CS-mPEG@Cur can achieve satisfactory antitumor effects in vivo.

We also monitored the mass of the mice every 3 days (Figure 8B). There was a slight increase in body mass observed after the administration of saline and $\mathrm{C}_{16}-\mathrm{SS}-\mathrm{CS}-\mathrm{mPEG}$ micelles showing that both micelles were biocompatible in vivo. A slight body mass loss was observed after the administration of $\mathrm{C}_{16}$-SS-CS-mPEG@Cur, which may have been due to the decreased mass of tumor tissue. In addition, the anti-inflammatory effects of $\mathrm{C}_{16}$-SS-CS-mPEG@, Cur or Cur against the tumor microenvironment were further verified by histological analyses of skin sections around tumors (Figure 8E). Compared with healthy control, tissues around tumors showed signs of severe inflammation, and massive numbers of inflammatory cells were observed. Cur treatment showed no remission of inflammation in the tumor microenvironment. In contrast, significantly reduced numbers of inflammatory cells were observed after being treated with $\mathrm{C}_{16}$-SS-CS-mPEG@Cur, indicating strong anti-inflammatory effect in vivo. This result may be because $\mathrm{C}_{16}-\mathrm{SS}-\mathrm{CS}$ mPEG@Cur treatment enhances Cur accumulation in tumor microenvironment and leads to raised Cur uptake of inflammatory cells. The improvement of inflammation in tumor microenvironment contributes to the inhibition of tumor, and it plays a synergistic role in the treatment process. ${ }^{3}$ Taken together, this confirmed that $\mathrm{C}_{16}$-SS-CS-mPEG@Cur micelles could be efficient nanocarriers for redox-responsive controlled drug delivery in vitro and tumor-targeted therapy in vivo.

\section{Conclusion}

An amphiphilic polymer $\mathrm{C}_{16}-\mathrm{SS}-\mathrm{CS}-\mathrm{mPEG}$ was synthesized by conjugating the hydrophilic $\mathrm{mPEG}$ and the hydrophobic alkyl chain (by a reduction-responsive disulfide bond) to CS. The polymer self-assembled into nanosize micellar form in aqueous solution. In vitro release studies revealed that $\mathrm{C}_{16}-\mathrm{SS}-\mathrm{CS}-\mathrm{mPEG} @$ Cur micelles were sensitive to a 
A

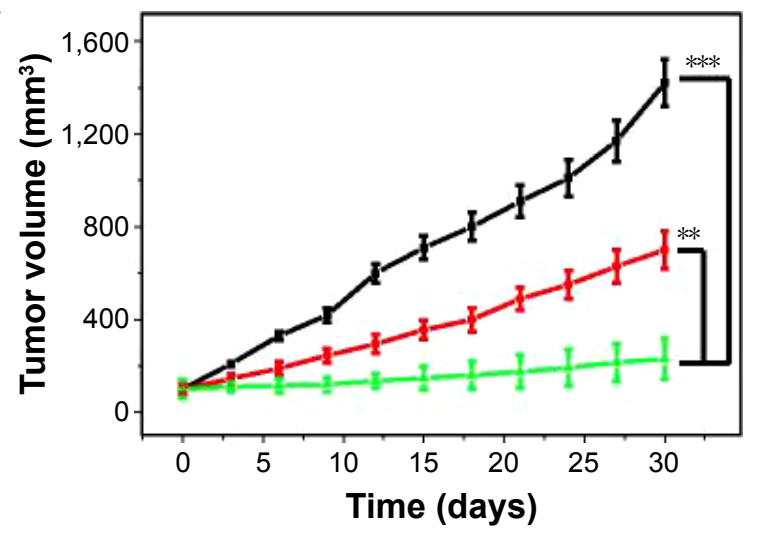

B

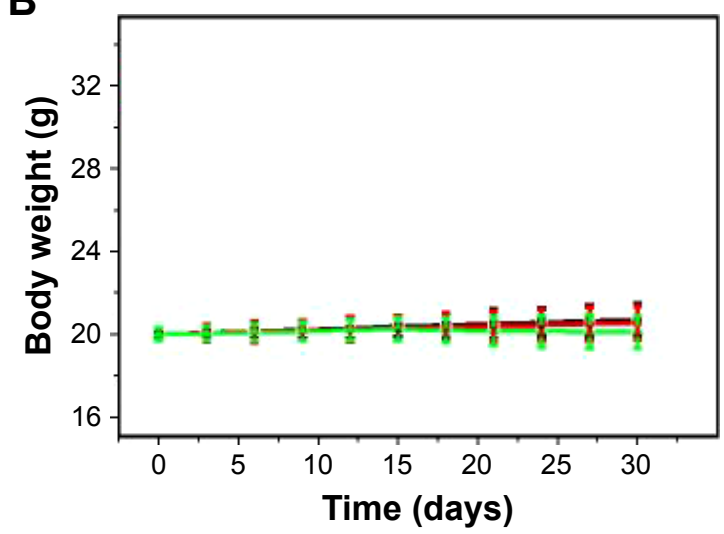

-"- Saline -*-Cur $=-\mathrm{C}_{16}$-SS-CS-mPEG@Cur

C

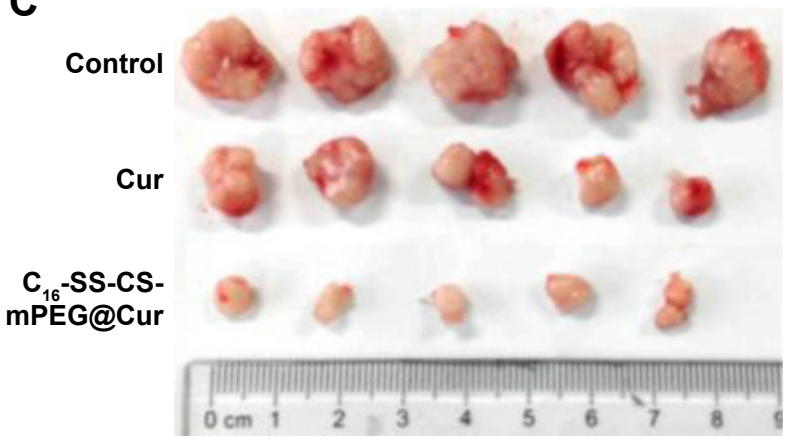

E
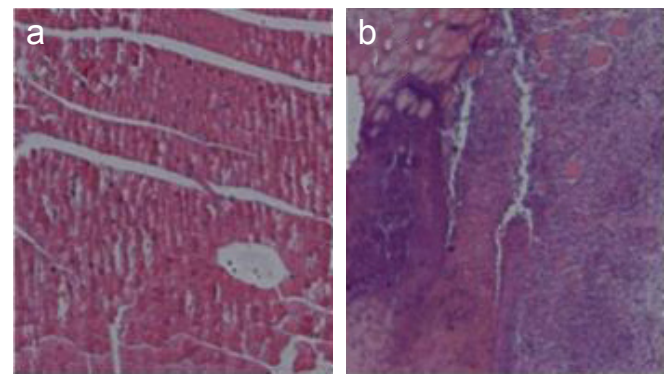
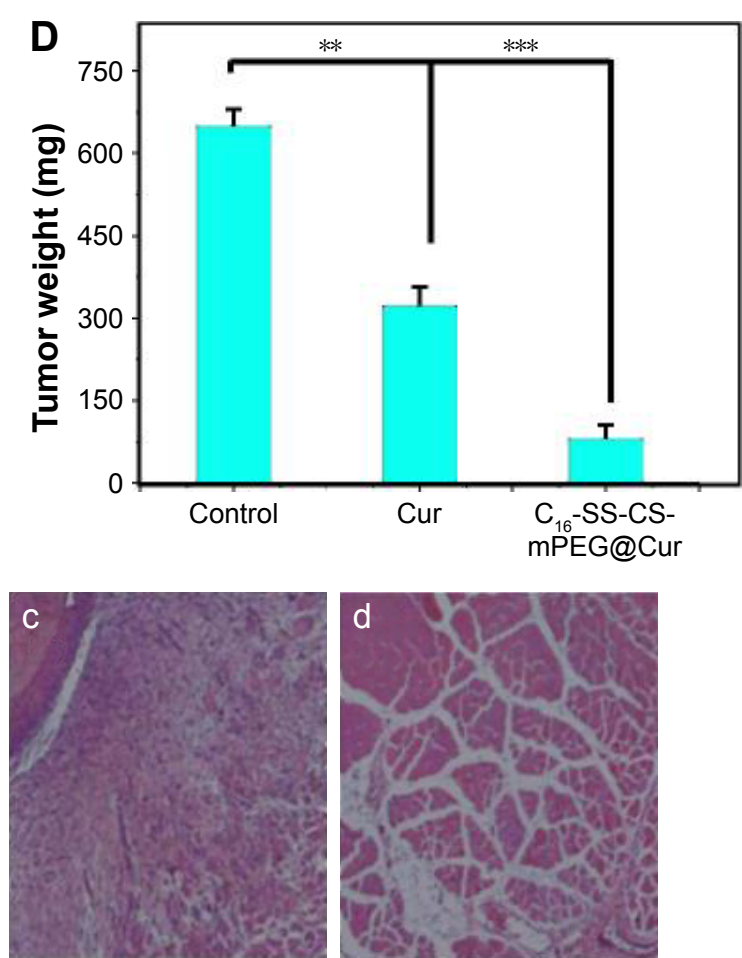

Figure 8 Anti-cancer and anti-inflammatory effects in vivo.

Notes: (A) Tumor volumes at different times after treatment. (B) The average mouse body mass was monitored using an electronic balance every 3 days. (C) The images of dislodged tumors from the mice 30 days posttreatment. (D) The mass of dislodged tumors from the mice in all five groups. $* * P<0.0 \mathrm{I}$, $* * * P<0.00 \mathrm{I}$. (E) Hematoxylin and eosin-stained images of skin sections around tumors collected from (a) healthy control, (b) saline, (c) Cur, and (d) $\mathrm{C}_{16}$-SS-CS-mPEG@Cur-injected mice bearing MCF-7 tumors 30 days postinjection. Data are shown as mean \pm SD $(n=5)$.

Abbreviations: $\mathrm{C}_{16}$-SS-CS-mPEG, monomethoxy-poly(ethylene glycol)-chitosan-S-S-hexadecyl; Cur, curcumin; SD, standard deviation.

reducing agent, such as DTT, which led to rapid drug release. Similar results were found by intracellular drug release assay; moreover, $\mathrm{C}_{16}$-SS-CS-mPEG@Cur efficiently enhanced cellular uptake of Cur, which led to the highest cytotoxicity. The anti-inflammatory effects of $\mathrm{C}_{16}$-SS-CS-mPEG@Cur have also been tested by measurement of the downregulated TNF- $\alpha$ expression. The result showed that $\mathrm{C}_{16}-\mathrm{SS}-\mathrm{CS}-$ mPEG@Cur exhibited a satisfactory anti-inflammatory effect and could improve the inflammation in tumor microenvironment. Furthermore, $\mathrm{C}_{16}$-SS-CS-mPEG@Cur administered via intravenous injection could significantly inhibit tumor growth in vivo and $\mathrm{C}_{16}$-SS-CS-mPEG@Cur benefited from its redox-responsive controlled intracellular release at the tumor site and synergistic effect of inflammation treatment. The present study affords a promising strategy for redoxresponsive drug delivery systems for cancer therapy.

\section{Acknowledgment}

This work was supported by the National Natural Science Foundation of China (21171070, 21371075), 
the Natural Science Foundation of Guangdong Province (2014A030311025, S2013010011660), and the Planned Item of Science and Technology of Guangdong Province (2016A020217011).

\section{Disclosure}

The authors report no conflicts of interest in this work.

\section{References}

1. Mantovani A, Allavena P, Sica A, Balkwill F. Cancer-related inflammation. Nature. 2008;454(7203):436-444.

2. Grivennikov SI, Greten FR, Karin M. Immunity, inflammation, and cancer. Cell. 2010;140(6):883-899.

3. Pollard J. Bacteria, inflammation and cancer. Nat Rev Immunol. 2015; 15(9):528-543.

4. Sica A, Allavena P, Mantovani A. Cancer related inflammation: the macrophage connection. Cancer Lett. 2008;267(2):204-215.

5. Coussens LM, Werb Z. Inflammation and cancer. Nature. 2002; 420(6917):860-867.

6. Marques JG, Gaspar VM, Costa E, Paquete CM, Correia IJ. Synthesis and characterization of micelles as carriers of non-steroidal antiinflammatory drugs (NSAID) for application in breast cancer therapy. Colloids Surf B Biointerfaces. 2014;113:375-383.

7. Ebrahim Attia AB, Oh P, Yang C, et al. Insights into EPR effect versus lectin-mediated targeted delivery: biodegradable polycarbonate micellar nanoparticles with and without galactose surface decoration. Small. 2014;10(21):4281-4286.

8. Lukyanov AN, Torchilin VP. Micelles from lipid derivatives of watersoluble polymers as delivery systems for poorly soluble drugs. Adv Drug Deliv Rev. 2004;56(9):1273-1289.

9. Xiao Y, Hong H, Javadi A, et al. Multifunctional unimolecular micelles for cancer-targeted drug delivery and positron emission tomography imaging. Biomaterials. 2012;33(11):3071-3082.

10. Heger M, van Golen RF, Broekgaarden M, Michel MC. The molecular basis for the pharmacokinetics and pharmacodynamics of curcumin and its metabolites in relation to cancer. Pharmacol Rev. 2014;66(1): 222-307.

11. Fan X, Zhang C, Liu D-B, Yan J, Liang H-P. The clinical applications of curcumin: current state and the future. Curr Pharm Des. 2013;19(11): 2011-2031.

12. Boyanapalli SS, Kong A-NT. "Curcumin, the King of Spices": epigenetic regulatory mechanisms in the prevention of cancer, neurological, and inflammatory diseases. Curr Pharmacol Rep. 2015;1(2):129-139.

13. Dhule SS, Penfornis P, He J, et al. The combined effect of encapsulating curcumin and C6 ceramide in liposomal nanoparticles against osteosarcoma. Mol Pharm. 2014;11(2):417-427.

14. Naksuriya O, Okonogi S, Schiffelers RM, Hennink WE. Curcumin nanoformulations: a review of pharmaceutical properties and preclinical studies and clinical data related to cancer treatment. Biomaterials. 2014;35:3365-3383.
15. Liang J, Wu W, Lai D, Li J, Fang C. Enhanced solubility and targeted delivery of curcumin by lipopeptide micelles. J Biomater Sci Polym Ed. 2015;26(6):369-383.

16. Preetha A, Ajaikumar BK, Robert AN, Bharat BA. Bioavailability of curcumin: problems and promises. Mol Pharm. 2007;4(6):807-818.

17. Kim TH, Jiang HH, Youn YS, et al. Preparation and characterization of water-soluble albumin-bound curcumin nanoparticles with improved antitumor activity. Int J Pharm. 2011;403(1-2):285-291.

18. Sarisozen C, Abouzeid AH, Torchilin VP. The effect of co-delivery of paclitaxel and curcumin by transferrin-targeted PEG-PE-based mixed micelles on resistant ovarian cancer in 3-D spheroids and in vivo tumors. Eur J Pharm Biopharm. 2014;88(2):539-550.

19. Huh KM, Lee SC, Cho YW, Lee J, Jeong JH, Park K. Hydrotropic polymer micelle system for delivery of paclitaxel. J Control Release. 2005; 101(1-3):59-68.

20. Yang Y, Xie X, Yang Y, et al. Polymer nanoparticles modified with photo- and ph-dual-responsive polypeptides for enhanced and targeted cancer therapy. Mol Pharm. 2016;13(5):1508-1519.

21. Hu FQ, Meng P, Dai YQ, et al. PEGylated chitosan-based polymer micelle as an intracellular delivery carrier for anti-tumor targeting therapy. Eur J Pharm Biopharm. 2008;70(3):749-757.

22. Maruyama K. Intracellular targeting delivery of liposomal drugs to solid tumors based on EPR effects. Adv Drug Deliver Rev. 2011;63(3): $161-169$.

23. Lee JH, Lee K, Moon SH, Lee Y, Park TG, Cheon J. All-in-one target-cell-specific magnetic nanoparticles for simultaneous molecular imaging and siRNA delivery. Angew Chem Int Ed Engl. 2009;48(23): 4174-4179.

24. Perumal OP, Inapagolla R, Kannan S, Kannan RM. The effect of surface functionality on cellular trafficking of dendrimers. Biomaterials. 2008;29(24-25):3469-3476.

25. Basnet P, Hussain H, Tho I, Skalko-Basnet N. Liposomal delivery system enhances anti-inflammatory properties of curcumin. J Pharm Sci. 2012;101(2):598-609.

26. Nasser MW, Elbaz M, Ahirwar DK, Ganju RK. Conditioning solid tumor microenvironment through inflammatory chemokines and S100 family proteins. Cancer Lett. 2015;365(1):11-22.

27. Giorgia DA, Gemma N, Chiara DM, Pietro M, Vladimir T. Gellan gum nanohydrogel containing anti-inflammatory and anti-cancer drugs: a multi-drug delivery system for a combination therapy in cancer treatment. Eur J Pharm Biopharm. 2014;87:208-216.

28. Cho J, Lee K, Kim C. Curcumin attenuates the expression of IL-1 $\beta$, IL-6, and TNF- $\alpha$ as well as cyclin E in TNF- $\alpha$-treated HaCaT cells; NF- $\mathrm{\kappa B}$ and MAPKs as potential upstream targets. Int J Mol Med. 2007;19(3): 469-474.

29. Xiao B, Laroui H, Ayyadurai S, Viennois E, Charania MA, Zhang Y. Mannosylated bioreducible nanoparticle-mediated macrophage-specific TNF-alpha RNA interference for IBD therapy. Biomaterials. 2013; 34(30):7471-7482. 


\section{Supplementary material}

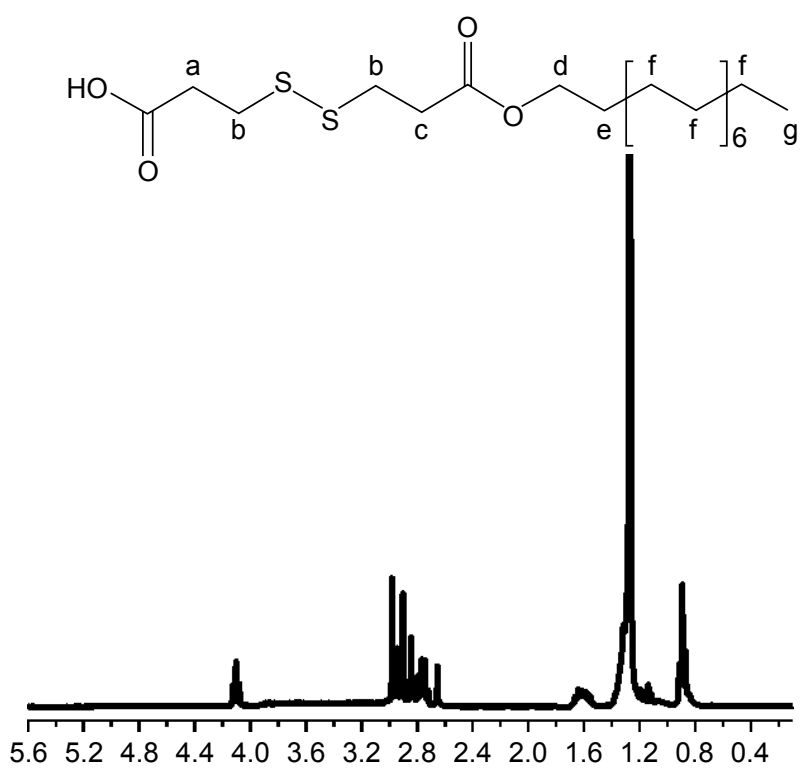

Figure $\mathrm{SI}$ ' $\mathrm{H}$ NMR spectra of $\mathrm{C}_{16}$-SS-COOH polymer in $\mathrm{D}_{2} \mathrm{O}$ (ppm).

Abbreviation: 'H NMR, 'H nuclear magnetic resonance.

\section{Publish your work in this journal}

The International Journal of Nanomedicine is an international, peerreviewed journal focusing on the application of nanotechnology in diagnostics, therapeutics, and drug delivery systems throughout the biomedical field. This journal is indexed on PubMed Central, MedLine, CAS, SciSearch $®$, Current Contents ${ }^{\circledR} /$ Clinical Medicine,
Journal Citation Reports/Science Edition, EMBase, Scopus and the Elsevier Bibliographic databases. The manuscript management system is completely online and includes a very quick and fair peer-review system, which is all easy to use. Visit http://www.dovepress.com/ testimonials.php to read real quotes from published authors. 